\title{
Computably Enumerable Sets that are Automorphic to Low Sets
}

\author{
Peter Cholak and Rachel Epstein
}

\begin{abstract}
We work with the structure consisting of all computably enumerable (c.e.) sets ordered by set inclusion. The question we will partially address is which c.e. sets are autormorphic to low (or low 2 ) sets. Using work of R. Miller 9, we can see that every set with semilow complement is $\Delta_{3}^{0}$ automorphic to a low set. While it remains open whether every set with semilow complement is effectively automorphic to a low set, we show that there are sets without semilow complement that are effectively automorphic to low sets. We also consider other lowness notions such as having a semilow 1.5 complement, having the outer splitting property, and having

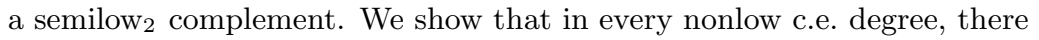
are sets with semilow 1.5 complements without semilow complements as well as sets with semilow 2 complements and the outer splitting property that do not have semilow 1.5 complements. We also address the question of which sets are automorphic to low 2 sets.
\end{abstract}

\section{Introduction}

Our domain of discourse is the collection of all c.e. sets under inclusion. This structure is called $\mathcal{E}$. By adding intersection, union, the empty set and $\omega$, this structure is a lattice. We should also mention that these binary operations in addition to $\emptyset, \omega$, and being computable and finite are definable in this structure. Thus, if we have an automorphism of $\mathcal{E}$ then computable sets must go to computable sets and infinite sets to infinite sets.

If we take the quotient of $\mathcal{E}$ modulo the ideal of finite sets, we get the lattice $\mathcal{E}^{*}$ of c.e. sets up to finite difference. Soare [13, XV 2.5] showed that if there is an automorphism of $\mathcal{E}^{*}$ taking $A$ to $B$, then there is one of $\mathcal{E}$ as well, so we can work in $\mathcal{E}^{*}$ to show that automorphisms of $\mathcal{E}$ exist.

A c.e. set $W$ is low if and only if its jump, $W^{\prime}$, is Turing equvialent to $\emptyset^{\prime}$. As we will discuss below, the low sets are special within $\mathcal{E}$. Our goal is to understand as best possible which sets look like and behave like low sets in this structure. That is, when does a set have a low set in its orbit?

The first result in this vein is a result of Soare 12 following [11. Soare showed that if a c.e. set $A$ is low then its collection of c.e. supersets of $A$ under inclusion is isomorphic to $\mathcal{E}$. Formally, $\mathcal{L}(A)=\left\{W_{e} \cup A \mid e \in \omega\right\}$ is isomorphic 
to $\mathcal{E}$. This suggests that low sets are similar to computable sets. Again, we can work modulo the finite sets, showing that the quotient of $\mathcal{L}(A)$ modulo the finite sets, called $\mathcal{L}^{*}(A)$, is isomorphic to $\mathcal{E}^{*}$.

Actually Soare proved a stronger result. But for that we need a few definitions. First a set $X$ is semilow if and only if $\left\{e \mid W_{e} \cap X \neq \emptyset\right\} \leq_{T} \mathbf{0}^{\prime}$. This index set is $\Sigma_{1}^{X}$. If $\bar{X}$ is c.e. and low then this index set is $\Delta_{2}^{0}$. So if $A$ is low then $A$ has a semilow complement. Now consider an isomorphism $\Phi$ between $\mathcal{L}^{*}(A)\left(\right.$ or $\left.\mathcal{E}^{*}\right)$ and $\mathcal{E}^{*}$. There are functions $g$ and $h$ such that $\Phi\left(W_{e} \cup A\right)=W_{g(e)}$ and $\Phi^{-1}\left(W_{e}\right)=W_{h(e)} \cup A$ (or $\Phi\left(W_{e}\right)=W_{g(e)}$ and $\left.\Phi^{-1}\left(W_{e}\right)=W_{h(e)}\right)$. If computable $\left(\Delta_{3}^{0}\right) g$ and $h$ can be found for $\Phi$, then we call $\Phi$ effective $\left(\Delta_{3}^{0}\right)$. It is standard to use the terms "effective" or " $\Delta_{3}^{0}$ " to describe isomorphisms between $\mathcal{L}(A)$ or $\mathcal{E}$ and $\mathcal{E}$, even though the isomorphisms produced may only be effective or $\Delta_{3}^{0}$ on the quotient spaces $\mathcal{L}^{*}(A)$ and $\mathcal{E}^{*}$. This is the way we will use these terms in this paper.

Soare [12 showed that if $A$ is c.e. set with semilow complement then $\mathcal{L}^{*}(A)$ is effectively isomorphic to $\mathcal{E}^{*}$. There have been several improvements on this result. We observe, using work of R. Miller [9], that this can be improved to if $A$ is a c.e. set with semilow complement then $A$ is $\Delta_{3}^{0}$ automorphic to a low set (see Section 2). This means there is a $\Delta_{3}^{0}$ automorphism $\Phi$ such that $\Phi(A)=\widehat{A}$ is low. One recent open question raised by Soare (personal communication) is whether the above $\Delta_{3}^{0}$ can be replaced by effective. Another question raised by Soare is to characterize the sets which are effectively automorphic to low sets. It was thought that perhaps a characterization would be all sets which have semilow complements. But, in Section 3, we show that this is not the case, as there are sets without semilow complements that are effectively automorphic to low sets.

Soare's result about sets with semilow complements was first improved by Maass 8. Maass showed that for any c.e. set $A$ with semilow 1.5 complements that $\mathcal{L}(A)$ is $\Delta_{3}^{0}$ isomorphic to $\mathcal{E}$. A set $X$ is semilow $w_{1.5}$ if and only if the index set $\left\{e \mid W_{e} \cap X\right.$ is finite $\} \leq_{1} \emptyset^{\prime \prime}$. By an index set argument, we know that if $A$ is effectively automorphic to a low set $\widehat{A}$ it must have semilow 1.5 complement $\left(W_{e} \cap A \neq^{*} \emptyset\right.$ if and only if $W_{g(e)} \cap \widehat{A} \neq^{*} \emptyset$ and if $\widehat{A}$ is low then the latter is $\Pi_{2}^{0}$ ).

Now Harrington and Soare [5, Section 5] show that there is a property $N L(A)$ definable in the structure $\mathcal{E}$ such that if $N L(A)$ holds then $A$ does not have semilow complement. Morever they showed that there is a set $A$ with semilow 1.5 complement and $N L(A)$. Thus we know that not all sets $A$ with semilow 1.5 complement can be automorphic to a low set. But can they be automorphic to $\mathrm{low}_{2}$ sets?

We thought we had a positive answer but $N L(A)$ for nonlow $2 A$ seems to be a barrier. $N L(A)$ does provide a barrier for the question about whether two promptly simple sets with semilow complements are automorphic. This barrier is discussed in Section 5.4 of [5]. (Our situation is similar. The issue seems to be that we cannot get $\widehat{A}$ to cover $A$ in real time. Even though we know a state $\nu$ is emptied into $A$, it is emptied slowly through a series of moves into RED and BLUE sets. On the $\widehat{A}$ side we have to match this series of BLUE and 
RED moves and hence we cannot quickly cover $A$. Another problem here is that depending on $e$ the series of RED and BLUE moves can change. The series of moves does not just depend on $\nu$.)

There was one more extension of Soare's and Maass's work. Cholak [1] showed that if $A$ has the outer splitting property and has semilow 2 complement then $\mathcal{L}(A)$ is isomorphic to $\mathcal{E}$. A has the outer splitting property if and only if there are computable functions $g$ and $h$ such that for all $e, W_{e}=W_{g(e)} \sqcup W_{h(e)}$, $W_{g(e)} \cap \bar{A}={ }^{*} \emptyset$ and if $W_{e} \cap \bar{A}$ is infinite then $W_{g(e)} \cap \bar{A}$ is nonempty. Maass [8. Lemma 2.3] shows in a very clever argument that if $\bar{A}$ is semilow 1.5 then $A$ has the outer splitting property. $X$ is semilow 2 if and only if the index set $\left\{e \mid W_{e} \cap X\right.$ is infinite $\} \leq_{T} \emptyset^{\prime \prime}$. At one time we thought we could show that

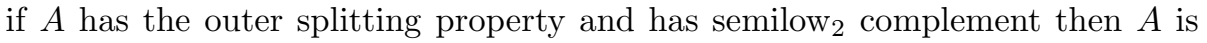
automorphic to a low 2 set. Note that if $A$ is automorphic to a low 2 then it has semilow $_{2}$ complement.

There is also some related recent work of Epstein, 4]. Epstein shows that there is a properly low 2 degree such that every c.e. set in that degree is automorphic to a low set. We were wondering if that could be shown more modularly. We wondered if there is some property $P$ such that every set with property $P$ is automorphic to a low set and there is some properly low 2 degree such that every set in that degree has property $P$. One reasonable candidate for $P$ would be having semilow complement. But Soare [13, IV 4.10] shows that every nonlow degree contains a c.e. set whose complement is not semilow (via a nice index set argument). Other later results rule out other possible $P$ 's.

Downey, Jockusch, and Schupp [3, Theorems 4.6 and 4.7] showed that every nonlow degree contains a c.e. $A$ without the outer splitting property (so $A$ 's complement is not semilow 1.5 ). In related results, we show that every nonlow degree contains a c.e. set $A$ whose complement is semilow 1.5 but not semilow (see Section (4) and a c.e. set $A$ whose complement is semilow 2 but not semilow 1.5 and has the outer splitting property (see Section 5). We also provide a nice index set argument that every nonlow 2 degree contains a c.e. set $A$ whose complement is not semilow 2 .

We should mention that it has been long known that if a degree is nonlow 2 it must contain a c.e. set which is not automorphic to a low 2 set. Lachlan [7] showed, using a true stages construction, that every low $_{2}$ set has a maximal superset while Shoenfield [10] showed that every nonlow 2 degree contains a c.e. set with no maximal superset. These two results have been generalized by Cholak and Harrington 2. One corollary of the work by Cholak and Harrington is that if $\mathbf{a}$ and $\mathbf{b}$ are two c.e. degrees and $\mathbf{a}^{\prime \prime} \not_{T} \mathbf{b}^{\prime \prime}$, then $\mathbf{a}$ contains a c.e. set not automorphic to anything computable from $\mathbf{b}$. It is open if this can be improved to show that a contains a c.e. set not automorphic to anything whose double jump is computable from $\mathbf{b}^{\prime \prime}$.

We mention one more open related question: if $A$ is low $_{2}$ then is $\mathcal{L}(A)$ isomorphic to $\mathcal{E}$ ? We now know that there is a properly low 2 set without the outer splitting property. Thus, a positive result here may not use the outer splitting property and is very likely to use the a true stage construction in the 
style of Lachlan [7].

\section{C.e. sets with semilow complements are auto- morphic to low}

Soare [12] showed that if $A$ is a c.e. set with semilow complement then $\mathcal{L}(A)$ is effectively isomorphic to $\mathcal{E}$. It has been conjectured that in fact any c.e. set $A$ with semilow complement is effectively automorphic to a low set. Here we show that it is possible to modify a proof of R. Miller $[9]$ to show that every c.e. set with semilow complement can be taken by a $\Delta_{3}^{0}$ automorphism to a low set.

Theorem 2.1. [R. Miller, [9], Theorem 1.1.1] For every c.e. set A with semilow complement and every noncomputable c.e. set $C$, there exists a $\Delta_{3}^{0}$ automorphism of $\mathcal{E}$ mapping $A$ to a set $B$ such that $C \not_{\mathrm{T}} B$.

R. Miller states this theorem differently, saying that $A$ is a low set instead of a set with semilow complement. However, he mentions that the construction only requires that $A$ have semilow complement.

We modify R. Miller's proof to get the following theorem.

Theorem 2.2. For every c.e. set $A$ with semilow complement, there exists a $\Delta_{3}^{0}$ automorphism of $\mathcal{E}$ mapping $A$ to a low set $B$.

Proof. Here we discuss the minor modifications to the proof of Theorem 2.1 that result in a proof of Theorem 2.2. Because our modifications are minor and the original proof using the complex Harrington-Soare automorphism construction of [6], we will not reproduce R. Miller's proof here. Instead we briefly sketch the proof and refer the reader of this section to Theorem 2.1] in [9] for more details.

To prove Theorem 2.1. R. Miller builds an automorphism on a tree, as in Harrington-Soare [6], which takes a given set $A$ to a constructed set $B$ with the desired property that $C \mathbb{E}_{\mathrm{T}} B$. The primary challenge of the theorem is to allow enough flow of elements into $B$ to match the flow of elements into $A$ while simultaneously restraining elements from $B$ so that $B$ cannot compute $C$. There are two key components of the construction. The first is a list $\mathcal{L}^{\mathcal{G}}$ that keeps track of the states of elements flowing into $A$ so that we can ensure that if infinitely many elements flow into $A$ in a given state, then infinitely many will flow into $B$ in the matching state. The second key component is Step $\hat{0}$, which enumerates elements into $B$ that are in the appropriate states. Step $\hat{0}$ only allows elements to enter if they are large enough to preserve a given restraint. In our construction, we modify $\mathcal{L}^{\mathcal{G}}$ and Step $\hat{0}$ to reflect our new restraint, but little else is changed.

In R. Miller's construction, Step 0 was the only step that involved putting elements into $B$. He needed to guarantee the proper flow of elements into $B$, while preserving restraint that would ensure that $B$ would not be able to compute $C$. In our modified construction, we just need to preserve a different restraint. In fact, this part of the construction can be done as in Step B of 
Epstein's proof in [4] that there is a nonlow degree such that every set in that degree is $\Delta_{3}^{0}$ automorphic to a low set.

In order to make this modification, we first must alter the list $\mathcal{L}^{\mathcal{G}}$. In $\mathrm{R}$. Miller's construction, pairs $\langle\alpha, \widehat{\nu}\rangle$ are added to the list whenever an element $x$ enters $A$ from the $\alpha$-state $\nu$. (Note that $\widehat{\nu}$ is the corresponding state on the $\bar{B} / B$ side of the construction.) In our modification, we will instead add the triple $\langle\alpha, \widehat{\nu}, x\rangle$ to the list $\mathcal{L}^{\mathcal{G}}$ whenever $x$ enters $A$ from the $\alpha$-state $\nu$. Note that we can identify each triple with a number in $\omega$.

Next, we replace R. Miller's Step $\hat{0}$ with the following new Step $\hat{0}$.

Step 0̂: (Moving elements into B.)

Find the first triple $\left\langle\alpha, \widehat{\nu}_{0}, x\right\rangle$ in $\mathcal{L}^{\mathcal{G}}$ such that there is a $\widehat{y} \in \widehat{\omega}$ that has never before caused action on this step satisfying all of the following:

(0.1) $\alpha$ is consistent;

(0.2) $\widehat{y} \in R_{\alpha, s}$;

(0.3) $\widehat{\nu}(\alpha, \widehat{y}, s)=\widehat{\nu}_{0}$; and

(0̂.4) for all $i<\left\langle\alpha, \widehat{\nu}_{0}, x\right\rangle, \varphi_{i}^{B}(i)[s] \downarrow \Longrightarrow \varphi_{i}^{B}(i)[s]<\widehat{y}$.

Action. If $\left\langle\alpha, \widehat{\nu}_{0}, x\right\rangle$ is not checked, check $\langle\alpha, x, e\rangle$, and do not enumerate $\widehat{y}$ into $B$. If $\left\langle\alpha, \widehat{\nu}_{0}, x\right\rangle$ has been checked already, enumerate $\widehat{y}$ into $B$ and remove $\left\langle\alpha, \widehat{\nu}_{0}, x\right\rangle$ from the list $\mathcal{L}^{\mathcal{G}}$. This will leave infinitely many elements in $\bar{B}$, while still matching the flow into $A$.

The purpose of this step is essentially the same as in the original construction. It creates a flow of elements into $B$ matching the flow into $A$, while also respecting the restraint of the negative requirements and ensuring that infinitely many elements remain outside of $B$.

Most of the lemmas in the proof of Theorem 2.1] in [9] could be kept exactly the same. Lemmas 3.1.3 and 3.3.4 would need only very minor and straightforward changes, reflecting how the new construction still guarantees that $\bar{B}$ is infinite and that Step 0 is able to enumerate an element for every $\left\langle\alpha, \widehat{\nu}_{0}, x\right\rangle$ on $\mathcal{L}^{\mathcal{G}}$, with $\alpha$ on the true path, which works essentially the same as before, but with the old restraint replaced by the new one.

The only significant difference in the verification would be to replace Lemmas 3.3.1 and 3.3.2 with the following lemma, which is the same as Lemma 8.17 in [4].

Lemma 2.3. The set $B$ is low.

Proof. Suppose there exist infinitely many $s$ such that $\Phi_{i}^{B}(i)[s] \downarrow$.

Let $s_{0}$ be the least $s$ such that for all $\langle\alpha, \widehat{\nu}, x\rangle \leq i,\langle\alpha, \widehat{\nu}, x\rangle$ has either been removed from the list $\mathcal{L}^{\mathcal{G}}$ already or will never be removed from the list (this can happen if it is never added to the list, or if it is added but never matched). Since each $\langle\alpha, \widehat{\nu}, x\rangle$ can enter $\mathcal{L}^{\mathcal{G}}$ only once, then after stage $s_{0}$, no $\widehat{y}$ will enter $B$ in order to match $\langle\alpha, \widehat{\nu}, x\rangle$. Let $s>s_{0}$ be some stage with $\Phi_{i}^{B}(i)[s] \downarrow$. Then by (0.4), nothing can enter $B$ below the use of this computation. So $(\forall t>s)$ $\left[\Phi_{i}^{B}(i)[t] \downarrow\right]$. So either $\left(\forall^{\infty} s\right)\left[\Phi_{i}^{B}(i)[s] \downarrow\right]$ or $\left(\forall^{\infty} s\right)\left[\Phi_{i}^{B}(i)[s] \uparrow\right]$. Thus, B is low. 
This completes the modification of R. Miller's proof to show that every c.e. set with semilow complement is $\Delta_{3}^{0}$ automorphic to a low set.

It remains open whether every c.e. set with semilow complement is effectively automorphic to a low set. In the next section, we show that there are sets without semilow complement that are effectively automorphic to low sets.

\section{Effectively automorphic to low but not semilow}

In Theorem 3.2, we build an effective automorphism of $\mathcal{E}^{*}$ that takes a set $A$ without semilow complement to a low set $\bar{A}$. To build an effective automorphism, we use Soare's Effective Extension Theorem.

The main tool in constructing an automorphism of $\mathcal{E}^{*}$ is matching infinite $e$ states. Suppose we are given listings of all the c.e. sets modulo finite difference, $\left\{U_{e}\right\}_{e \in \omega}$ and $\left\{V_{e}\right\}_{e \in \omega}$, and an invertible map $\Theta$ of $\mathcal{E}^{*}$ that takes $U_{e}$ to $\widehat{U}_{e}$ and $\Theta^{-1}$ takes $V_{e}$ to $\widehat{V}_{e}$. The $e$-state of an element $x$ tells us which c.e. sets $U_{i}$ and $\widehat{V}_{i}$, or $\widehat{U}_{i}$ and $V_{i}$ contain the element $x$, for all $i \leq e$. The map $\Theta$ is an automorphism of $\mathcal{E}^{*}$ if there are infinitely many elements in an $e$-state $\nu$ with respect to $U_{i}$ and $\widehat{V}_{i}$ if and only if there are infinitely many elements in the corresponding $e$-state $\widehat{\nu}$ with respect to $\widehat{U}_{i}$ and $V_{i}$.

More formally, we consider two copies of $\omega$, which we will refer to as $\omega$ and $\widehat{\omega}$. We imagine that our automorphism is given by a permutation of $\omega$, which can be represented as a function from $\omega$ to $\widehat{\omega}$. The $e$-state of an element $x \in \omega$ at stage $s$ is given by the triple $\nu(e, x, s)=\langle e, \sigma(e, x, s), \tau(e, x, s)\rangle$, where $\sigma(e, x, s)=\left\{i \leq e \mid x \in U_{i, s}\right\}$ and $\tau(e, x, s)=\left\{i \leq e \mid x \in \widehat{V}_{i, s}\right\}$. The $e$-state of an element $\widehat{x} \in \widehat{\omega}$ is determined the same way, except with $\widehat{U}_{i, s}$ replacing $U_{i, s}$ and $V_{i, s}$ replacing $\widehat{V}_{i, s}$. The final $e$-state of an element is $\nu(e, x)=\langle e, \sigma(e, x), \tau(e, x)\rangle$, where $\sigma(e, x)=\left\{i \leq e \mid x \in U_{i}\right\}$ and $\tau(e, x)=\left\{i \leq e \mid x \in \widehat{V}_{i}\right\}$, and similarly for $\widehat{x} \in \widehat{\omega}$.

To see that $\Theta$ is an automorphism, it suffices to show that

$$
\begin{aligned}
& (\forall \nu)\left(\exists^{\infty} x \in \omega\right)\left[\nu(e, x)=\nu \text { w.r.t. }\left\{U_{n}\right\}_{n \in \omega} \text { and }\left\{\widehat{V}_{n}\right\}_{n \in \omega}\right] \\
& \Longleftrightarrow\left(\exists^{\infty} \hat{y} \in \hat{w}\right)\left[\nu(e, \hat{y})=\nu \text { w.r.t. }\left\{\widehat{U}_{n}\right\}_{n \in \omega} \text { and }\left\{V_{n}\right\}_{n \in \omega}\right] .
\end{aligned}
$$

The theorem stated below is actually a special case of Soare's Extension Theorem [1974]. The full version is stronger than is needed for this paper. Recall that for an given enumeration of two c.e. sets $U$ and $V, U \backslash V=\{x \mid \exists s[x \in$ $\left.\left.\left(U_{s+1}-V_{s}\right)\right]\right\}, U$ before $V$ and $U \searrow V=(U \backslash V) \cap V, U$ before $U$ and then $V$.

Theorem 3.1 (The Extension Theorem). (Soare[1974]) Let $A$ and $\widehat{A}$ be infinite c.e. sets, and let $\left\{U_{n}\right\}_{n \in \omega},\left\{V_{n}\right\}_{n \in \omega},\left\{\widehat{U}_{n}\right\}_{n \in \omega}$, and $\left\{\widehat{V}_{n}\right\}_{n \in \omega}$ be computable arrays of c.e. sets. Suppose there is a simultaneous computable enumeration of all of the above such that $\widehat{A} \searrow \widehat{U}_{n}=\emptyset=A \searrow \widehat{V}_{n}$ for all $n$. For each full e-state $\nu$, define 
$D_{\nu}^{A}=\left\{x: x \in A_{a t s+1}\right.$ and $\nu=\nu(e, x, s)$ w.r.t. $\left\{U_{n, s}\right\}_{n, s \in \omega}$ and $\left.\left\{\widehat{V}_{n, s}\right\}_{n, s \in \omega}\right\}$

and

$D_{\widehat{\nu}}^{\widehat{A}}=\left\{\widehat{x}: \widehat{x} \in \widehat{A}_{a t s+1}\right.$ and $\widehat{\nu}=\nu(e, \widehat{x}, s)$ w.r.t. $\left\{\widehat{U}_{n, s}\right\}_{n, s \in \omega}$ and $\left.\left\{V_{n, s}\right\}_{n, s \in \omega}\right\}$.

$D_{\nu}^{A}$ is the set of all elements that enter $A$ from e-state $\nu$, and similarly for $D_{\widehat{\nu}}^{\widehat{T}}$.

Suppose that the simultaneous enumeration satisfies:

$$
(\forall \nu)\left[D_{\nu}^{A} \text { infinite } \Longleftrightarrow D_{\widehat{\nu}}^{\widehat{A}} \text { infinite }\right] .
$$

Then there is a computable enumeration of c.e. sets $\left\{\widetilde{U}_{n}\right\}_{n \in \omega}$ and $\left\{\widetilde{V}_{n}\right\}_{n \in \omega}$, where $\widetilde{U}_{n}$ extends $\widehat{U}_{n}$ and $\widetilde{V}_{n}$ extends $\widehat{V}_{n}$ such that $\widehat{U}_{n}=\widetilde{U}_{n} \backslash \widehat{A}, \widehat{V}_{n}=\widetilde{V}_{n} \backslash A$, and

$$
\begin{gathered}
\exists^{\infty} x \in A \text { with final e-state } \nu \text { w.r.t. }\left\{U_{n, s}\right\}_{n, s \in \omega} \text { and }\left\{\widetilde{V}_{n, s}\right\}_{n, s \in \omega} \\
\Longleftrightarrow \\
\exists^{\infty} \widehat{x} \in \widehat{A} \text { with final e-state } \widehat{\nu} \text { w.r.t. }\left\{\widetilde{U}_{n, s}\right\}_{n, s \in \omega} \text { and }\left\{V_{n, s}\right\}_{n, s \in \omega} .
\end{gathered}
$$

A skeleton of the c.e. sets is a simultaneous computable enumeration of c.e. sets such that every c.e. set is finitely different from some set on the list. Let $\left\{U_{n}\right\}_{n \in \omega}$ and $\left\{V_{n}\right\}_{n \in \omega}$ be skeletons of the c.e. sets. Thus, if we begin with a partial automorphism of $\mathcal{E}^{*}$ on the complements of $A$ and $\widehat{A}$ that takes $U_{n}$ to $\widehat{U}_{n}$ and $\widehat{V}_{n}$ to $V_{n}$, then we can extend it to an automorphism of $\mathcal{E}^{*}$ that takes $A$ to $\widehat{A}, U_{n}$ to $\widetilde{U}_{n}$, and $\widetilde{V}_{n}$ to $V_{n}$.

The way we build a partial automorphism on the complements of $A$ and $\widehat{A}$ is to match infinite $e$-states for elements in $\bar{A}$ and $\bar{A}$. That is, if there are infinitely many elements $x \in \bar{A}$ such that $\nu(e, x)=\nu$, then there are infinitely many elements in $\widehat{y} \in \overline{\widehat{A}}$ such that $\widehat{\nu}(e, \widehat{y})=\widehat{\nu}$, and vice versa.

We call the state $\nu(\widehat{\nu})$ a gateway state if $D_{\nu}^{T}\left(D_{\widehat{\nu}}^{\widehat{T}}\right)$ is infinite. In the full version of the Extension Theorem, we do not need an exact matching of gateway states $\nu$ and $\widehat{\nu}$, but only a covering of the states, as described in Soare [1974]. Our construction gives an exact matching of gateway states, so we have stated only the special case of the theorem here.

Theorem 3.2 (Cholak/Epstein). There is a set A that does not have semilow complement, but is effectively automorphic to a low set.

Proof. We will construct a c.e. set $\bar{A}$ that is not semilow and a c.e. set $\widehat{A}$ that is low, such that $A$ and $\widehat{A}$ are effectively automorphic to each other. We use Soare's Effective Extension Theorem. 


\subsection{Requirements}

Our first requirement is the automorphism requirement, which constructs an automorphism taking $A$ to $\widehat{A}$. To do this, we build a simultaneous computable enumeration of c.e. sets $\left\{U_{n}\right\}_{n \in \omega},\left\{V_{n}\right\}_{n \in \omega},\left\{\widehat{U}_{n}\right\}_{n \in \omega}$, and $\left\{\widehat{V}_{n}\right\}_{n \in \omega}$ as in the extension theorem, such that $\left\{U_{n}\right\}_{n \in \omega}$ and $\left\{V_{n}\right\}_{n \in \omega}$ are skeletons. We will ensure that the map taking $U_{n}$ to $\widehat{U}_{n}$ and $\widehat{V}_{n}$ to $V_{n}$ gives a partial automorphism of $\mathcal{E}^{*}$ on the complements of $A$ and $\widehat{A}$, and that we have equality of gateway states to meet the hypotheses of the Extension Theorem.

Let $\varphi_{i}$ be a listing of all partial computable functions of two variables. We will use it to list all $\Delta_{2}$ functions.

To achieve that $A$ does not have semilow complement, we meet the following requirement for all $i \in \omega$ :

$\mathrm{P}_{i}: \quad \widehat{\varphi}_{i}(e):=\lim _{s} \varphi_{i}(e, s)$ is not the characteristic function of $\left\{e: W_{e} \cap \bar{A}\right.$ nonempty\}.

To this end, for each $i \in \omega$ we will build sets $S_{k}^{i}$ for each $k \in\left\{0,1, \ldots 4^{i+1}\right\}$, such that for some $k, S_{k}^{i}$ will be the c.e. set $W_{e}$ and $\widehat{\varphi}_{i}(e)=\lim _{s} \varphi_{i}(e, s)$ will be wrong about whether $W_{e}$ and $\bar{A}$ have nonempty intersection. The index $e$ for $S_{k}^{i}$ may be found computably in $k, i$, and the number of times $n$ we have started over in building $S_{k}^{i}$. We call this computable function $g(i, k, n)$. By the Recursion Theorem with Parameters (see [13, II 3.5]), we may assume that we know the function $g$ in advance. We can rewrite $\mathrm{P}_{i}$ as:

$\mathrm{P}_{i}: \quad(\exists k)(\exists n)\left[0 \leq k \leq 4^{i+1}\right.$ and $\widehat{\varphi}_{i}(g(i, k, n))=1 \Longrightarrow S_{k}^{i} \cap \bar{A}=\emptyset$, and $\widehat{\varphi}_{i}(g(i, k, n))=0 \Longrightarrow S_{k}^{i} \cap \bar{A} \neq \emptyset$, for $S_{k}^{i}$ having been reset $n$ times].

The number $4^{i+1}$ appears as it is the number of $i$-states. Since each $i$-state is determined by a subset of $\left\{U_{0}, \ldots, U_{i}\right\}$ as well as a subset of $\left\{\widehat{V}_{0}, \ldots, \widehat{V}_{i}\right\}$, there are $2^{i+1} \cdot 2^{i+1}=4^{i+1}$. This will be important for matching entry states, as we will see in the next section.

To achieve that $\widehat{A}$ is low, we meet the usual requirement for all $j \in \omega$ :

$\mathrm{N}_{j}:\left(\exists^{\infty} s\right) \Phi_{j}^{\widehat{A}}(j)[s] \downarrow \Longrightarrow \Phi_{j}^{\widehat{A}}(j) \downarrow$.

This guarantees that $\widehat{A}$ is low because it makes the jump of $\widehat{A}$ limit computable, and thus computable in $\mathbf{0}^{\prime}$.

\subsection{Basic strategy}

Our construction will take place on two identical pinball machines, $M$ and $\widehat{M}$, see Cholak [1, Soare [13] or Harrington and Cholak [6. Each element of $\omega$ will flow through $M$, and each element of a copy of $\omega$ called $\widehat{\omega}$ will flow through $\widehat{M}$. The construction of the pinball machines is extremely simple. They each have a single corridor along which all elements flow. Along the corridor are gates $G_{e}$ for each $e \in \omega$. Elements may be held at gates or pass through, according to the construction. In our construction, a closed gate will let nothing through, while an open gate will let all elements through except for those currently designated as witness, as explained later. Throughout the construction, we will match elements in $\omega$ to elements in $\widehat{\omega}$ by a matching function $m(x)$ with domain $\omega$, 
where we begin with $m(x)=\widehat{x}$, the copy of $x$ in $\widehat{\omega}$. During the construction, elements may be rematched.

Let $\left\{W_{e}\right\}_{e \in \omega}$ be a standard enumeration of the c.e. sets. We build two skeletons $\left\{U_{e}\right\}_{e \in \omega}$ and $\left\{V_{e}\right\}_{e \in \omega}$ as follows: If $x \in W_{e}$ and $x$ is either at gate $G_{e^{\prime}}$ for $e^{\prime} \geq e$ or $x$ has been removed from $M$, enumerate $x$ into $U_{e}$. Similarly, if $x \in W_{e}$ and $\widehat{x}$ is either at gate $G_{e^{\prime}}$ for $e^{\prime} \geq e$ or $\widehat{x}$ has been removed from $\widehat{M}$, enumerate $\widehat{x}$ into $V_{e}$. For each $e$, only finitely many elements never reach gate $G_{e}$ or leave the machines, so $U_{e}={ }^{*} W_{e}$ and $V_{e}={ }^{*} W_{e}$.

Elements move through $M$ by flowing through the machine, starting at gate $G_{0}$, then moving to $G_{1}$, and so on. Every element on the machine is at a gate. As $x$ moves, $m(x)$ copies its move on $\widehat{M}$. To meet the automorphism requirement, if $x$ is in $U_{e}$ while at gate $G_{e^{\prime}}$ for $e^{\prime} \geq e$, enumerate $m(x)$ into $\widehat{U}_{e}$. Similarly, if $m(x)$ is in $V_{e}$, enumerate $x$ into $\widehat{V}_{e}$.

We define for each stage $s$ a restraint function

$$
r(j, s)=\max \left\{\varphi_{j^{\prime}}^{\widehat{A}}\left(j^{\prime}\right)[s] \mid j^{\prime} \leq j\right\} .
$$

That is, $r(j, s)$ is the maximum use of any jump computation $\Phi_{j^{\prime}}^{\widehat{A}}\left(j^{\prime}\right)[s]$ for $j^{\prime} \leq j$.

To meet $\mathrm{P}_{i}$, we would like to build a c.e. set $S$ such that if $\varphi_{i}$ guesses that $S \cap \bar{A}$ is empty, we put an element not currently in $A$ into $S$, and if it later guesses that $S \cap \bar{A}$ is nonempty, we put that element into $A$. This is the standard method of constructing a set that does not have semilow complement. It is also frequently used to build a nonlow set, as any set without semilow complement is not low. In order to meet the automorphism requirement, if we put infinitely many elements into $A$, we must put infinitely elements into $\widehat{A}$ from the same $i$-state. We cannot simply enumerate $\widehat{x}$ into $\widehat{A}$ whenever we enumerate $x$ into $A$ because we need to ensure that $\widehat{A}$ is low. Instead, when we put an element into $A$, we would like to put a large enough element into $\widehat{A}$ that is in the same $i$-state. The way we will accomplish this is to build several c.e. sets $S_{k}^{i}$ for each $i$ and we will guarantee that one will act as the desired set $S$. When we want to enumerate an element $x$ from some $S_{k_{0}}^{i}$ into $A$ to satisfy a positive requirement, we will simultaneously enumerate a large enough element $\widehat{z}$ into $\widehat{A}$, where $\widehat{z}$ is in the same $i$-state as $x$. Since we have removed $x$ and $\widehat{z}$ from the machines, we have left $m(x)$ and $m^{-1}(\widehat{z})$ without reasonable partners, and so we partner them with each other. By this process, whenever we enumerate an element into $A$ from some $i$-state, we also enumerate an element into $\widehat{A}$ from the same $i$-state, so that we achieve exact matching of entry states, as desired by the Effective Extension Theorem.

For every $i$, we build $S_{k}^{i}$ for each $k \in\left\{0,1, \ldots 4^{i+1}\right\}$. We will start with all sets empty. If $\varphi_{i}$ guesses that they all have empty intersection with $\bar{A}$, then we will close gate $G_{i}$ and begin to fill each $S_{k}^{i}$ with a single element, in increasing order of $k$. We fill $S_{k}^{i}$ with the least element $x$ at gate $G_{i}$ such that $m(x)>r(j, s)$ for $j=i+k$ and $m(x)$ is greater than any element in any $S_{k^{\prime}}^{i}$ for $k^{\prime}<k$.

When a set $S_{k}^{i}$ contains an element not in $A$, we call that element $x$ and its 
partner $m(x)$ witnesses. Once each $S_{k}^{i}$ contains an element, we reopen the gate $G_{i}$ to all non-witnesses.

We then wait until $\varphi_{i}$ guesses that each $S_{k}^{i}$ has nonempty intersection with $\bar{A}$. Now, there are $4^{i+1}+1$ witnesses and only $4^{i+1}$ different $i$-states. Thus, by the pigeonhole principle, there are two witnesses in the same $i$-state. Say they are $x_{0}$ in $S_{k_{o}}^{i}$ and $x_{1}$ in $S_{k_{1}}^{i}$, with $k_{0}<k_{1}$. We will enumerate $x_{0}$ into $A$ and $m\left(x_{1}\right)$ into $\widehat{A}$. These elements are entering in the same state. Once elements enter $A$ or $\widehat{A}$, they are removed from the pinball machine. Their previous matches, $m\left(x_{0}\right)=\widehat{y}$ and $x_{1}$, do not get removed from the pinball machine. Instead, set $m\left(x_{1}\right)$ to be $\widehat{y}$, the element previously known as $m\left(x_{0}\right)$. We reset all $S_{k^{\prime}}^{i}$ for $k^{\prime}>k_{0}$, removing the name "witness" from any elements in sets that are reset, and starting new empty $S_{k^{\prime}}^{i}$. Note that $S_{k_{0}}^{i}$ has not been reset, but its only element has entered $A$.

If ever $\varphi_{i}$ again guesses correctly which sets $S_{k}^{i}$ have nonempty intersection with $\bar{A}$, then we must repeat the process, with some minor changes. We again close the gate $G_{i}$ until there is a single element in each $S_{k}^{i}$ that is not also in $A$. For $k=0$, we fill $S_{0}^{i}$ as before, with the least element $x$ such that $m(x)>r(j, s)$ for $j=i+0=i$. For $k>0$, we wait until $S_{k-1}^{i}$ has been filled and enumerate into it the least $x$ such that $m(x)>r(j, s)$ for $j=i+k+n_{k}^{i}$, where $n_{k}^{i}$ is the number of times $S_{k}^{i}$ has been reset by the action of $\mathrm{P}_{i}$. The purpose of $n_{k}^{i}$ is so that if $\mathrm{P}_{i}$ acts infinitely often, it will still only injure each $\mathrm{N}_{j}$ finitely often. Once each $S_{k}^{i}$ contains a witness, we reopen $G_{i}$ to all non-witnesses. We then continue as in the previous paragraph.

In order that $S_{k}^{i}$ respect $\mathrm{N}_{j}$ whenever $j \leq i+k+n_{k}^{i}$, if $\Phi_{j}^{\widehat{A}}(j)[s+1] \downarrow$ by a new computation, we reset $S_{k}^{i}$. This causes any witnesses to no longer be witnesses and the set to be built again from an empty set. Note that it does not cause $n_{k}^{i}$ to increase because the resetting was caused by $\mathrm{N}_{j}$ and not by the action of $\mathrm{P}_{i}$.

The primary reason for using multiple $S_{k}^{i}$ sets instead of a single $S^{i}$ is that we want to respect more and more computations when we enumerate elements into $\widehat{A}$, which we could not do with only a single $S^{i}$ that we can only reset finitely often. We will show in the verification that there will be some $S_{k}^{i}$ that is only reset finitely often that we will use to satisfy $P_{i}$.

\subsection{Construction}

Stage $s=0$. Let each $n_{k}^{i}=0$. All sets begin empty and all gates begin open.

Stage $s+1$.

Step 1: Place $s$ on machine $M$ at gate $G_{0}$ and $\widehat{s}$ on machine $\widehat{M}$ at gate $G_{0}$. Let $m(s)=\widehat{s}$.

Step 2: For each $x$ and $e$, if $x \in W_{e, s}$, and $x$ is either at gate $G_{e^{\prime}}$ for $e^{\prime} \geq e$ or $x$ has been removed from the machine, enumerate $x$ into $U_{e, s+1}$. Similarly, if $\widehat{x} \in W_{e, s}$, and either $\widehat{x}$ is at gate $G_{e^{\prime}}$ for $e^{\prime} \geq e$ or $\widehat{x}$ has been removed from the machine, enumerate $\widehat{x}$ into $V_{e, s+1}$.

In addition, if $x \in U_{e, s+1}$ and is still on the machine, enumerate $m(x)$ into 
$\widehat{U}_{e, s+1}$. Similarly, if $\widehat{x} \in V_{e, s+1}$ and is still on the machine, enumerate $m^{-1}(\widehat{x})$ into $\widehat{V}_{e, s+1}$.

Step 3: If $\Phi_{j}^{\widehat{A}}(j)[s] \downarrow$ via a new computation, reset all $S_{k}^{i}$ with $k+i+n_{k}^{i} \geq j$ by creating a new $S_{k}^{i}$, increasing $n_{k}^{i}$ by one, and cancelling all witnesses from $S_{k}^{i}$.

Step 4: For each $i$, in increasing order, check if either of the following cases apply.

Case $4 A$ (filling $S_{k}^{i}$ ): At least one $S_{k}^{i}$ has empty intersection with $\bar{A}$, and either the gate is closed or $\varphi_{i}\left(g\left(i, k, n_{k}^{i}\right), s\right)$ is equal to the characteristic function of $S_{k}^{i} \cap \bar{A}$ for each $k$. Close the gate if not already closed. For the least $k$ such that $S_{k}^{i} \cap \bar{A}$ has empty intersection, check if there is an $x$ at gate $G_{i}$ such that both $x$ and $m(x)$ are larger than any current or previous witness at the gate and $m(x)>r(j, s)$ for $j=i+k+n_{k}^{i}$. If so, enumerate $x$ into $S_{k}^{i}$ and call it and $m(x)$ witnesses. If we enumerated an element into $S_{k}^{i}$ for $k=4^{i+1}$, open the gate. Continue to Step 5.

Case $4 B$ (enumerating into $A$ and $\widehat{A}$ ): All $S_{k}^{i}$ have nonempty intersection with $\bar{A}$ and $\varphi_{i}\left(g\left(i, k, n_{k}^{i}\right), s\right)=1$ for all $k$. Find the least witness $x_{0}$ such that there is a witness $x_{1}$ in the same $i$-state as $x_{0}$. Such a pair is guaranteed by the pigeonhole principle. Say the element $x_{0}$ is in $S_{k_{0}}^{i}$ and $x_{1}$ is in $S_{k_{1}}^{i}$. Note that $k_{0}<k_{1}$. Enumerate $x_{0}$ into $A$ and $m\left(x_{1}\right)$ into $\widehat{A}$ and remove both $x_{0}$ and $m\left(x_{1}\right)$ from the machines. Now $x_{1}$ and $\widehat{y}=m\left(x_{0}\right)$ do not have matches on the machine, so set $m\left(x_{1}\right)=\widehat{y}$. Reset each $S_{k}^{i}$ for $k>k_{0}$ by increasing $n_{k}^{i}$ and starting $S_{k}^{i}$ over with no witnesses. Note that $S_{k_{0}}^{i}$ does not get reset and so is not empty, but now has empty intersection with $\bar{A}$ and thus no witnesses.

Step 5: For each $x$ at an open gate $G_{e}$ such that $x$ is not a witness and $x, m(x)>e$, move $x$ and $m(x)$ to gate $G_{e+1}$ on their respective machines.

\subsection{Verification}

Lemma 3.3. Each $N_{j}$ is injured finitely often and is satisfied, so $\widehat{A}$ is low.

Proof. Induct on $j$. Suppose true for all $j^{\prime}<j$. Then since $\mathrm{N}_{j^{\prime}}$ is injured finitely often, $\lim _{s} r\left(j^{\prime}, s\right)$ exists.

If $\Phi_{j}^{\widehat{A}}(j)[s] \downarrow$, then the only $S_{k}^{i}$ that can injure the computation by enumerating $m(x)$ into $\widehat{A}$ for $x \in S_{k}^{i}$ must satisfy $i+k<j$. Each of these $S_{k}^{i}$ gets reset finitely often by $\mathrm{N}_{j^{\prime}}$, for $j^{\prime}<j$, by the induction hypothesis. By the construction, after an element $m(x)$ enters $\widehat{A}, S_{k}^{i}$ gets reset and $n_{k}^{i}$ increases by one, as $S_{k}^{i}$ is playing the role of $S_{k_{1}}^{i}$ in the construction, where $k_{1}>k_{0}$. Thus, after finitely many elements of the form $m(x)$ with $x \in S_{k}^{i}$ enter $\widehat{A}$, any future elements $x \in S_{k}^{i}$ must satisfy $m(x)>r(j, s)$ since $i+k+n_{k}^{i}>j$ for large enough $n_{k}^{i}$. Since $\mathrm{N}_{j}$ can only be injured finitely often by finitely many $S_{k}^{i}, \mathrm{~N}_{j}$ will eventually be satisfied. Thus, the jump of $\widehat{A}$ is limit computable, so $\widehat{A}$ is low. 
Lemma 3.4. For each gate $G_{i}$, infinitely many elements pass through gate $G_{i}$ to gate $G_{i+1}$.

Proof. Induct on $i$. Suppose true for all $i^{\prime}<i$. Then infinitely many elements reach gate $G_{i}$.

Note that if gate $G_{i}$ is ever closed, then it will reopen when an element enters $S_{k}^{i}$ for $k=4^{i+1}$. First we show that it will eventually reopen. As $G_{i}$ is closed, we know that $S_{4^{i+1}}^{i}$ has empty intersection with $\bar{A}$, so Case $4 \mathrm{~A}$ applies. While the gate is closed, Case 4B will not act for this $i$, so the only way for any $S_{k}^{i}$ to be reset is for $\Phi_{j}^{\widehat{A}}(j)[s]$ to converge via a new computation for $j \leq k+i+n_{k}^{i}$. However, no $n_{k}^{i}$ will change for any $k$ while $G_{i}$ is closed, and Lemma 3.3 tells us that $\Phi_{j}^{\widehat{A}}(j)[s]$ can only converge via a new computation finitely often, so each $S_{k}^{i}$ will only be reset finitely often while $G_{i}$ remains closed. Furthermore, for $j=i+k+n_{k}^{i}, r(j, s)$ will eventually stop increasing while the gate remains closed. As infinitely many elements arrive at gate $G_{i}$, each $S_{k}^{i}$ will eventually receive an element, including $S_{4^{i+1}}^{i}$, at which point the gate is opened. Therefore, whenever gate $G_{i}$ is closed, it is eventually reopened.

If the gate $G_{i}$ is closed only finitely often, then almost all elements that enter $G_{i}$ also enter $G_{i+1}$. Consider the case that $G_{i}$ is closed infinitely often. Then it is opened infinitely often, meaning that infinitely often, all $S_{k}^{i}$ have nonempty intersection with $\bar{A}$. In order for $G_{i}$ to become closed again, some $S_{k}^{i}$ must have empty intersection with $\bar{A}$. This can only happen by Step 4 Case $4 \mathrm{~B}$ or Step 3 applies. In either case, there must be some $S_{k}^{i}$ that gets reset and so its witness is no longer a witness, and on Step 5, it moves to gate $G_{i+1}$ if large enough. Thus, infinitely often, an element moves from $G_{i}$ to $G_{i+1}$.

Lemma 3.5. For each e, $W_{e}={ }^{*} U_{e}={ }^{*} V_{e}$, and thus $\left\{U_{e}\right\}_{e \in \omega}$ and $\left\{V_{e}\right\}_{e \in \omega}$ are skeletons.

Proof. We will prove that $W_{e}={ }^{*} U_{e}$. The proof that $W_{e}={ }^{*} V_{e}$ is similar. Note that $U_{e} \subseteq W_{e}$, as we never enumerate an element into $U_{e}$ until after it appears in $W_{e}$. Note also that the only elements in $W_{e}$ that are not enumerated into $U_{e}$ are those that are permanently held at a gate $G_{i}$ for $i<e$. We know from Lemma 3.4 that all gates are open at infinitely many stages, thus no element is held forever at gate $G_{i}$ unless it is forever labeled a witness or if either $x$ or $m(x)$ are less than or equal to $i$. The latter situation happens for finitely many elements. When any $S_{k}^{i}$ is reset, its witnesses are cancelled, meaning they are no longer considered to be witnesses, so any element that is forever a witness is in the final incarnation of $S_{k}^{i}$ and never enters $A$. (For the $V_{e}$ case, these witnesses are of the form $m(x)$ for $x \in S_{k}^{i} \cap \bar{A}$.) There is never more than one element of $S_{k}^{i} \cap \bar{A}$, for each $i<e$, so there are finitely many elements that are forever kept at $G_{i}$ as witnesses. Thus, all other elements of $W_{e}$ eventually either leave the machine or reach $G_{e}$ and are enumerated into $U_{e}$.

Lemma 3.6. Requirement $P_{i}$ is met, so $\bar{A}$ is not semilow. 
Proof. We must show that $\lim _{s} \varphi_{i}(e, s) \neq\left\{e \mid W_{e} \cap \bar{A} \neq \emptyset\right\}$. Suppose for a contradiction that it is false. Let $i$ be the least such that $\lim _{s} \varphi_{i}(e, s)$ gives the characteristic function of the set of $e$ such that $W_{e}$ has nonempty intersection with $\bar{A}$.

Note that $S_{0}^{i}$ can only be reset by $\mathrm{N}_{j}$ for $j \leq i$, which will only reset $S_{0}^{i}$ finitely often. Let $k^{*}$ be the greatest $k$ such that $S_{k}^{i}$ is only ever reset finitely often. If $k^{*}=4^{i+1}$, then Step 4 only acts finitely often, meaning that Case $4 \mathrm{~A}$ and Case 4B only apply finitely often, so for almost all $s, \varphi_{i}$ guesses wrong about whether some $S_{k}^{i}$ has nonempty intersection with $\bar{A}$. Thus, since we are assuming that $\varphi_{i}$ is eventually correct, we cannot have that $k^{*}=4^{i+1}$.

Now, let $S_{k^{*}}^{i}$ be reset $n$ times in total. Then $\lim _{s} \varphi_{i}\left(g\left(i, k^{*}, n\right), s\right)$ is 1 if $S_{k^{*}}^{i}$ has nonempty intersection with $\bar{A}$ and 0 otherwise. Since $k^{*} \neq 4^{i+1}, S_{k^{*}+1}^{i}$ gets reset infinitely often, which means that Step 4 Case 4B acts infinitely often by enumerating an element $x \in S_{k^{*}}^{i}$ into $A$. Thus, infinitely often, $S_{k^{*}}^{i}$ gets a new witness in Case $4 \mathrm{~A}$ and then has that witness enumerated into $A$ in Case 4B. Therefore, there are infinitely many stages when $S_{k^{*}}^{i}$ has empty intersection with $\bar{A}$ and Case $4 \mathrm{~A}$ applies because $\varphi_{i}\left(g\left(i, k^{*}, n\right), s\right)=0$ and there are infinitely many stages when $S_{k^{*}}^{i}$ has nonempty intersection with $\bar{A}$ and Case 4B applies because $\varphi_{i}\left(g\left(i, k^{*}, n\right), s\right)=1$. This contradicts the assumption that $\lim _{s} \varphi_{i}\left(g\left(i, k^{*}, n\right), s\right)$ exists. Therefore, requirement $\mathrm{P}_{i}$ is met, and since $\left\{e \mid W_{e} \cap \bar{A} \neq \emptyset\right\}$ is not limit computable, $\bar{A}$ is not semilow.

Lemma 3.7. There is an effective automorphism of $\mathcal{E}^{*}$ taking $A$ to $\widehat{A}$.

Proof. Note that the construction provides a simultaneous computable enumeration of the sets $\left\{\widehat{U}_{e}\right\}_{e \in \omega}$ and $\left\{\widehat{V}_{e}\right\}_{e \in \omega}$. We guarantee that $\nu$ is infinite on the complement of $A$ if and only if $\widehat{\nu}$ is infinite on the complement of $\widehat{A}$ by always enumerating elements simultaneously with their partners: $x$ and $m(x)$ are in the same $e$-state, where $G_{e}$ is the highest gate they reach. Note that there is no possibility for either $x$ or $\widehat{x}$ to be rematched infinitely often, as they each only reach finitely many gates and can only be witnesses once at each gate. Also note that if $x$ and $m(x)$ are permanently partners and remain forever at gate $G_{e}$, then they are in the same $e^{\prime}$-state for all $e^{\prime}>e$ as well, since neither are enumerated into $U_{i}, V_{i}, \widehat{U}_{i}$ or $\widehat{V}_{i}$.

Next, we apply the Effective Extension Theorem. It suffices to show that the gateway states for $A$ equal the gateway states for $\widehat{A}$. We only ever enumerate into either $A$ or $\widehat{A}$ during Step 4 Case 4B. When we do, we pick witnesses $x_{0}$ and $x_{1}$ at Gate $G_{i}$ that are in the same $i$-state, which are guaranteed to exist by the pigeonhole principle. Then, we enumerate $x_{0}$ into $A$ and $m\left(x_{1}\right)$ into $\widehat{A}$. If $x_{0}$ is in $i$-state $\nu$, then $m\left(x_{1}\right)$ is in $\widehat{\nu}$. As above, note that $x_{0}$ and $m\left(x_{1}\right)$ are also in the same $e$-state for any $e$ because if $e>i$, then neither element is in $U_{e}, V_{e}, \widehat{U}_{e}$, or $\widehat{V}_{e}$. Thus, we get that the gateway states $D_{\nu}^{A}$ equal the gateway states $D_{\widehat{\nu}}^{\widehat{A}}$.

By the Effective Extension Theorem, we can extend the partial automorphism on the complements of $A$ and $\widehat{A}$ to a total automorphism of $\mathcal{E}^{*}$ taking $A$ 
to $\widehat{A}$.

\section{Semilow $_{1.5}$ not semilow in all nonlow degrees}

In Theorem 3.2 we showed that there is set $A$ that does not have semilow complement and is effectively automorphic to a low set. Since $A$ is effectively automorphic to a low set, $A$ has semilow 1.5 complement (see the introduction).

In Theorem 4.1, we show that in every nonlow c.e. degree, there is a set that has semilow 1.5 complement but that does not have semilow complement. It remains open whether we can find such sets in every degree that are also effectively automorphic to low sets.

Theorem 4.1. For every nonlow c.e. degree $\mathbf{d}$, there is a c.e. set $A \in \mathbf{d}$ such that $A$ has semilow $1_{1.5}$ complement, but does not have semilow complement.

Corollary 4.2. The nonlow c.e. degrees are precisely the degrees of c.e. sets that have semilow $w_{1.5}$ complement but not semilow complement.

The corollary follows immediately from the theorem, since every low c.e. set has semilow complement.

Proof. Given a nonlow c.e. set $D$, we will construct an $A \equiv_{\mathrm{T}} D$ such that $\bar{A}$ is semilow 1.5 but not semilow.

\subsection{Requirements:}

To ensure that $D \leq_{\mathrm{T}} A$, we will code $D$ into $A$. To begin, we construct a computable list of disjoint finite sets $F_{k} \subset \omega$, with $F_{0}$ containing 1 element and each other $F_{k}$ having $2 k^{2}$ elements.

$\mathrm{C}_{k}: k \in D$ if and only if some element of $F_{k}$ is in $A$.

To make $\bar{A}$ not semilow, we proceed similarly as in Theorem 3.2 by meeting the following requirement for each total computable function $h$ on two inputs:

$\mathrm{P}_{h}: \lim h(e, s)$ is not the characteristic function of $\left\{e \mid W_{e} \cap \bar{A} \neq \emptyset\right\}$.

Let $\left\{{ }^{s} \varphi_{i}\right\}_{i \in \omega}$ be a listing of all partial computable functions on two inputs. Then we must meet $\mathrm{P}_{h}$ for all $h=\varphi_{i}$. We find it easier to refer to $h$ instead of $i$ in this situation. However, when we say $h<j$, please note that if we are using $h$ to stand in for $\varphi_{i}$, then we mean that $i<j$.

We will try to meet $\mathrm{P}_{h}$ for each $\alpha$ of length $i$ for $h=\varphi_{i}$ on our tree of strategies, and for each $e \in \omega$, so we will label our requirements $\mathrm{P}_{h, e}^{\alpha}$. To meet $\mathrm{P}_{h}^{\alpha}$, for each $e$, if $\mathrm{P}_{h, e}^{\alpha}$ has been reset $n$ times, then we build $S_{h, e}^{\alpha}=W_{r(\alpha, e, n)}$, where $r$ is known in advance by the Recursion Theorem with Parameters. If $h$ guesses that $S_{h, e}^{\alpha} \cap \bar{A}$ is empty, we add an element, called a witness, to $S_{h, e}^{\alpha}$. If $h$ changes its guess, then we want to enumerate the witness into $A$. When $\mathrm{P}_{h, e}^{\alpha}$ gets reset, its witnesses become inactive. We will meet the requirement for some $e$ and $\alpha$, so that $W_{r(\alpha, e, n)} \cap \bar{A}$ is nonempty if and only if $\lim _{s} h(r(\alpha, e, n), s) \neq 1$. 
Define $Y_{h}=\left\{x \mid x\right.$ is ever a witness for some $\mathrm{P}_{h, e}^{\alpha}$, for any $e$ and $\left.\alpha\right\}$ and $Z_{h}=\left\{x \mid x\right.$ is a witness in $Y_{h}$ that becomes inactive but does not enter $\left.A\right\}$. Let $Y^{j}$ be defined as $\bigcup_{h \leq j} Y_{h}$ and $Z^{j}$ be defined as $\bigcup_{h \leq j} Z_{h}$. Thus $Y^{j}$ is the set of witnesses for nodes of length at most $j$ and $Z^{j}$ is the subset of $Y^{j}$ of witnesses that become inactive.

For $A$ to have semilow 1.5 complement, we need to ensure:

L: $\left\{j \mid W_{j} \cap \bar{A}\right.$ infinite $\} \leq_{m}$ Inf.

To do this, we will show that $W_{j} \cap \bar{A}$ is infinite if and only if either $W_{j} \cap Z^{j}$ is infinite or $W_{j}-\left(Y^{j} \cup A\right)$ has infinitely many expansionary stages, where a stage is expansionary if $\left(W_{j}-\left(Y^{j} \cup A\right)\right)[s]$ is larger than it has been at any previous stage. Since $W_{j} \cap Z^{j}$ and the set of expansionary stages of $W_{j}-\left(Y^{j} \cup A\right)$ are both c.e. sets, this will give us the desired $m$-reduction.

As mentioned, we will work on a binary tree of strategies. Let $\alpha$ be a length $i$ string on the tree. For each $j<i, \alpha(j)=0$ indicates a guess that $W_{j}-\left(Y^{j} \cup A\right)$ has infinitely many expansionary stages and $\alpha(j)=1$ is a guess that it has finitely many. $\mathrm{P}_{h}^{\alpha}$ will work to satisfy $\mathrm{P}_{h}$ while assuming that $\alpha$ is correctly guessing the true outcome.

The tree allows us to ensure that if $W_{j}-\left(Y^{j} \cup A\right)$ has infinitely many expansionary stages, it is in fact an infinite set. The nodes guessing that there are infinitely many expansionary stages are not allowed to add witnesses when $\mathrm{L}_{j}$ is injured. Instead, they wait until a new expansionary stage is reached. This way, they do not create witnesses that may later enter $W_{j}-\left(Y^{j} \cup A\right)$, only to be forced into $A$. In addition, nodes guessing that $W_{j}-\left(Y^{j} \cup A\right)$ has finitely many expansionary stages are reset each time $W_{j}-\left(Y^{j} \cup A\right)$ reaches a new expansionary stage, meaning that any of their current witnesses will never enter $A$. The combination of these two actions guarantees that if $W_{j}-\left(Y^{j} \cup A\right)$ has infinitely many expansionary stages, it has infinitely many elements that never enter $A$ and so it is infinite.

In addition, we need that $A \leq_{\mathrm{T}} D$, which we achieve by a nonlow permitting argument using a technique of Downey, Jockusch, and Schupp [3. An element only becomes a witness for $\mathrm{P}_{h, e}^{\alpha}$ when $\Phi_{e}^{D}(e)[s] \downarrow$. If this computation later converges, such a witness gets enumerated into $A$. For each $\alpha$, we build a computable function on two inputs, $\ell_{\alpha}(e, s)$. If $\mathrm{P}_{h, e}^{\alpha}$ wishes to enumerate a witness into $A$ at stage $s$, we set $\ell_{\alpha}(e, s)=1$. Otherwise $\ell_{\alpha}(e, s)=0$. If the computation $\Phi_{e}^{D}(e)[s]$ is injured, then $D$ permits the witness to enter $A$ and so the witness enters $A$ as desired. We will only enumerate witnesses when permitted, so that we will achieve $A \leq_{\mathrm{T}} D$. Thus, we must ensure that $D$ permits frequently enough. Since $D$ is not low and $\ell_{\alpha}$ is computable, we know that $K^{D}(e)$ is not the limit of $\ell_{\alpha}(e, s)$ as $s$ approaches infinity, else $K^{D}$ would be limit computable and thus $D$ would be low. Therefore, there must be some $e$ such that $K^{D}(e) \neq \lim _{s} \ell_{\alpha}(e, s)$. When $\mathrm{P}_{h, e}^{\alpha}$ wishes to enumerate an element, $\ell_{\alpha}(e, s)=1$, so $\Phi_{e}^{D}(e)$ must diverge. When the current computation is injured by a new element entering $D$ below the use, $\mathrm{P}_{h, e}^{\alpha}$ has permission to enumerate the element into $A$. Thus, for each $\alpha$ on the true path, there is some $e$ such 
that $D$ will give permission for $\mathrm{P}_{h, e}^{\alpha}$ to enumerate elements into $A$ as often as needed.

\subsection{Construction}

For each $k$, we define a finite set $F_{k}$. Each of these sets will be contained within $\omega^{[0]}$, where $\omega^{[n]}$ is the $n$th "row" of $\omega$, the set of all elements of the form $\langle x, n\rangle$ using the standard pairing function. We let $F_{0}$ be the singleton set $\{\langle 0,0\rangle\}$, and for $k>0$, we let $F_{k}$ be the least $2 k^{2}$ elements in $\omega^{[0]}$ that have not appeared in any $F_{k^{\prime}}$ for $k^{\prime}<k$.

We build an approximation $\delta_{s}$ to the true path. We call $s$ an $\alpha$-stage if $\alpha$ is a substring of $\delta_{s}$. Define $\delta_{0}=\lambda$, the empty string. Let $\delta_{s}$ be the length $s$ string defined by: $\delta_{s}(j)=0$ if $W_{j}-\left(Y^{j} \cup A\right)$ has reached a new expansionary stage since the last $\delta_{s}\lceil j$-stage, and 1 otherwise.

Let $\ell_{\alpha}(e, 0)=0$ and $\ell_{\alpha}(e, s+1)=\ell_{\alpha}(e, s)$ unless otherwise specified. We begin with all $\mathrm{L}_{j}$ allowing addition of new witnesses for every $\mathrm{P}_{h}^{\alpha}$. During the construction, in order to prevent further injury to itself, $\mathrm{L}_{j}$ may disallow some $\mathrm{P}_{h}^{\alpha}$ from adding new witnesses. However, we will show that if $\alpha$ is on the true path, the construction ensures that $\mathrm{P}_{h}^{\alpha}$ will be allowed to add witnesses infinitely often.

Nodes on the tree may be put in 1-1 correspondence with $\mathbb{N}-\{0\}$ via a function $f: 2^{<\omega} \rightarrow \mathbb{N}-\{0\}$. Using such a correspondence, let $\langle\alpha, e\rangle:=\langle f(\alpha), e\rangle$. In Step 3, witnesses come from $\omega^{[\langle\alpha, e\rangle]}$.

Stage $s=0: \ell_{\alpha}(e, 0)=0$ and all sets $S_{h, e}^{\alpha}$ begin empty for all $\alpha, h$, and $e$.

Stage $s+1$ :

Step 1 (new expansionary stage): For each $j \in \omega$, if $W_{j}-\left(Y^{j} \cup A\right)$ grows to a size we haven't seen before, i.e. it reaches a new expansionary stage, then do the following for each $\alpha$ of length greater than $j$ : If $\alpha$ guesses that $W_{j}-\left(Y^{j} \cup A\right)$ has infinitely many expansionary stages (i.e. $\left.\alpha(j)=0\right)$, then $\mathrm{L}_{j}$ now allows addition of new witnesses for $\mathrm{P}_{h}^{\alpha}$. If $\alpha$ guesses that $W_{j}-\left(Y^{j} \cup A\right)$ has finitely many expansionary stages $(\alpha(j)=1)$, we reset $\mathrm{P}_{h, e}^{\alpha}$ for all $e$, making $\ell_{\alpha}(e, s)=0$ and declaring $\mathrm{P}_{h, e}^{\alpha}$ and any witnesses for $\mathrm{P}_{h, e}^{\alpha}$ inactive. When reset, we will start a new $S_{h, e}^{\alpha}$ that begins empty. This will ensure in Lemma 4.3 that nodes guessing incorrectly that $W_{j}-\left(Y^{j} \cup A\right)$ has finitely many expansionary stages will not prevent $W_{j} \cap \bar{A}$ from being infinite by putting too many elements into $A$.

Step 2 (allowing some disallowed witnesses): We say a node of length $j$ is allowed new witnesses if no $j^{\prime}<j$ disallows $\mathrm{P}_{h}^{\alpha}$ from adding witnesses. For each $j, N$, and $\beta$, where $\beta$ has length $j$, if $\mathrm{L}_{j}$ currently disallows for $N$ (as defined in Step 5) and if $\beta$ is allowed new witnesses, then set $\mathrm{L}_{j}$ to allow new witnesses for all $\alpha$ unless Step 2 has acted at some previous stage for these particular $j$, $N$, and $\beta$. Do this for all possible triples, of which there are only finitely many. This step guarantees that nodes on the true path will be allowed to act in Step 3 infinitely often.

Step 3 (adding witnesses): We act for each $\alpha$ of length at most $s$ such that $\mathrm{P}_{h}^{\alpha}$ is allowed to add witnesses by each $\mathrm{L}_{j}$ with $j<s$ such that $\alpha(j)=0$, and 
such that either $s$ is an $\alpha$-stage or $\alpha$ is to the left of $\delta_{s}$ and if $s_{\alpha}$ was the last $\alpha$-stage, $\mathrm{P}_{h}^{\alpha}$ has not been allowed to act at any stage $t, s_{\alpha} \leq t<s$. In other words, we act on the approximation to the true path $\delta_{s}$ and for any nodes to the left of $\delta_{s}$ that have not been allowed to act since before they were last on the approximation to the true path. We act in length-lexicographic order. For each such $\alpha$, we ask if there is any $e<s$ such that

- $\mathrm{P}_{h, e}^{\alpha}$ is inactive,

- $\Phi_{e}^{D}(e)[s] \downarrow$,

- $h(r(\alpha, e, n))=0$ for $n$ the number of times $\mathrm{P}_{h, e}^{\alpha}$ has been reset, and

- for all $e^{\prime}<e, \Phi_{e^{\prime}}^{D}\left(e^{\prime}\right)[s] \downarrow \Longleftrightarrow \ell_{\alpha}\left(e^{\prime}, s\right)=1$ or $\Phi_{e^{\prime}}^{D}\left(e^{\prime}\right)[s] \downarrow$ and has changed computations at least $e$ times since the last time that $\mathrm{P}_{h, e}^{\alpha}$ added a witness.

If so, then for each such $e$, let $x>s, x \in \omega^{[\langle\alpha, e\rangle]}$, be a fresh witness, larger than any previously chosen witness. Enumerate $x \in S_{h, e}^{\alpha}$. Declare $x$ to have use $s$, which we note is larger than the use of $\Phi_{e}^{D}(e)[s]$. We call both $\mathrm{P}_{h, e}^{\alpha}$ and $x$ active.

Step 4 (changing $\ell_{\alpha}$ when $h$ changes its guess): If $\mathrm{P}_{h, e}^{\alpha}$ is active, $\ell_{\alpha}(e, s)=0$, and $h(r(\alpha, e, n), s+1)=1$ where $n$ is the number of times $\mathrm{P}_{h, e}^{\alpha}$ has been reset, then set $\ell_{\alpha}(e, s+1)=1$. Reset $\mathrm{P}_{h, e^{\prime}}^{\alpha}$ for all $e^{\prime}>e$ by making $\mathrm{P}_{h, e^{\prime}}^{\alpha}$ and all elements in $S_{h, e^{\prime}}^{\alpha}$ inactive and starting new empty sets $S_{h, e^{\prime}}^{\alpha}$, as well as setting $\ell_{\alpha}\left(e^{\prime}, s+1\right)=0$. Perform this step for each $\alpha$ and for the least $e$ such that it applies.

Step 5 (enumerating witnesses into A and disallowing new witnesses): If $x \in S_{h, e}^{\alpha}$ is active with use $u$ and $D_{s+1}\left\lceil u \neq D_{s}\left\lceil u\right.\right.$, then put $x$ into $A_{s+1}$ and set $\ell_{\alpha}(e, s+1)=0$. Declare $\mathrm{P}_{h, e}^{\alpha}$ and $x$ inactive.

If by such an enumeration, we cause an element of $\left(W_{j}-\left(Y^{j} \cup A\right)\right)[s]$ to enter $A$, then we say $\mathrm{L}_{j}$ does not allow $\mathrm{P}_{h}^{\alpha}$ to add witnesses for any $\alpha$ such that $\alpha(j)=0$. In addition, if the size of $\left(W_{j}-\left(Y^{j} \cup A\right)\right)$ has reached the number $N$, we say that $\mathrm{L}_{j}$ disallows for $N$.

Step 6 (coding D): If $k \in D_{s+1}-D_{s}$, enumerate one element from $F_{k}$ into $A_{s+1}$ such that for each $M>0$, if $j+M=k$, then the element is not one of the least $M$ elements in $\left(W_{j}-\left(Y^{j} \cup A\right)\right)[s]$.

\subsection{Verification}

Lemma 4.3. For each $j \in \omega$, if the set of expansionary stages of $W_{j}-\left(Y^{j} \cup A\right)$ is infinite, then $W_{j} \cap \bar{A}$ is infinite.

Proof. Assume the set of expansionary stages of $W_{j}-\left(Y^{j} \cup A\right)$ is infinite. We will show that for each $M, W_{j}-\left(Y^{j} \cup A\right)$ has at least $M$ elements.

No element in $\left(W_{j}-\left(Y^{j} \cup A\right)\right)[s]$ ever enters $Y^{j}$ since new witnesses are chosen to be larger than $s$, and elements of $\left(W_{j}-\left(Y^{j} \cup A\right)\right)[s]$ are smaller than $s$. 
Induct on $M$. Assume true for $M-1$.

For $k \leq j$, only finitely many elements are ever put into $A$ by $\mathrm{C}_{k}$. For $k>j$, $\mathrm{C}_{k}$ can only bring the size of $W_{j}-\left(Y^{j} \cup A\right)$ below $M$ if $M+j>k$, which happens finitely often. Let $s_{0}$ be a stage by which the least $M-1$ elements in $\left(W_{j}-\left(Y^{j} \cup A\right)\right)\left[s_{0}\right]$ never enter $A$ and after which no $\mathrm{C}_{k}$ enumerates any of the least $M$ elements of $\left(W_{j}-\left(Y^{j} \cup A\right)\right)[s]$ into $A$. Let $s_{1}>s_{0}$ be a stage after which Step 2 never acts for $(j, M-1, \beta)$ for any $\beta$ of length $j$. We may assume that at every stage $s>s_{1}$, the $M$ th least element of $\left(W_{j}-\left(Y^{j} \cup A\right)\right)[s]$ is a witness for some $\mathrm{P}_{h, e}^{\alpha}$, else it would never enter $A$ and we would be guaranteed to have at least $M$ elements in $W_{j}-\left(Y^{j} \cup A\right)$, as desired. Note that the length of $\alpha$ is greater than $j$ since the witnesses are not in $Y^{j}$.

Let $s_{2}>s_{1}$ be a stage such the $M$ th least element in $\left(W_{j}-\left(Y^{j} \cup A\right)\right)\left[s_{2}\right]$, called $x_{M}$, is the least that will ever be in the $M$ th position of any $\left(W_{j}-\left(Y^{j} \cup\right.\right.$ $A))[s]$ for $s \geq s_{2}$. Now, if $x_{M}$ never enters $A$ by Step 5 , we are done, so assume $x_{M}$ enters $A$. When $x_{M}$ enters $A$, all larger witnesses also enter $A$, and since the next element in the $M$ th position is a witness, and it cannot be less than $x_{M}$ due to minimality, then the next element that enters the $M$ th position has yet to become a witness. When $x_{M}$ enters $A, \mathrm{~L}_{j}$ does not allow any $\mathrm{P}_{h}^{\alpha}$ to add witnesses for any $\alpha$ such that $\alpha(j)=0$. Step 2 has finished acting for $j$ and $M-1$, so it will not cause $\mathrm{L}_{j}$ to later allow. The next time $\mathrm{L}_{j}$ allows will be by Step 1, which means $\left(W_{j}-\left(Y^{j} \cup A\right)\right)$ has already reached a new expansionary stage. Thus, at the first expansionary stage after $x_{M}$ enters $A$, the only witness that could be in the $M$ th position must be a witness for an $\alpha$ with $\alpha(j)=1$. At that new expansionary stage, Step 1 inactivates the witnesses for $\alpha$. Thus the element in the $M$ th position can never enter $A$, so $\left(W_{j}-\left(Y^{j} \cup A\right)\right)$ has at least $M$ elements.

We say that $\alpha$ is on the true path if $\alpha(j)=0$ if and only if $W_{j}-\left(Y^{j} \cap \bar{A}\right)$ has infinitely many expansionary stages during the construction.

Lemma 4.4. Assume $\alpha$ is on the true path. Let $e$ be the least such that $\lim _{s} \ell_{\alpha}(e, s) \neq K^{D}(e)$. Then $P_{h, e}^{\alpha}$ is reset finitely often ( $n$ times) and $\lim _{s} h(r(\alpha, e, n), s)=$ $0 \Longleftrightarrow W_{r(\alpha, e, n)} \cap \bar{A}$ is nonempty. Thus, $\bar{A}$ is not semilow.

Proof. $\mathrm{P}_{h, e}^{\alpha}$ can only be reset in two ways: In Step 1 by $W_{j}-\left(Y^{j} \cup A\right)$ growing while $\alpha$ is guessing it has finitely many expansionary stages, which can only happen finitely often for the correct $\alpha$, and by Step 4 acting for $\mathrm{P}_{h, e^{\prime}}^{\alpha}$, for $e^{\prime}<e$. Such a $\mathrm{P}_{h, e^{\prime}}^{\alpha}$ only resets $\mathrm{P}_{h, e}^{\alpha}$ when $\ell_{\alpha}\left(e^{\prime}, s\right)$ changes to 1 from 0 . This can only happen finitely often since $\lim _{s} \ell_{\alpha}\left(e^{\prime}, s\right)$ must exist for $e^{\prime}<e$. Thus, $\mathrm{P}_{h, e}^{\alpha}$ is reset $n$ times for some finite $n$.

Case 1: Suppose $S_{h, e}^{\alpha} \cap \bar{A}$ is nonempty, for $S_{h, e}^{\alpha}=W_{r(\alpha, e, n)}$.

Then there is some $x \in S_{h, e}^{\alpha} \cap \bar{A}$ that was enumerated into $S_{h, e}^{\alpha}$ by Step 3 at stage $s_{0}+1$ when $\Phi_{e}^{D}(e)\left[s_{0}\right] \downarrow$ and $\ell_{\alpha}\left(e, s_{0}\right)=0$. We want to show that $\lim _{s} h(r(\alpha, e, n), s)=0$. 
Assume $h(r(\alpha, e, n), t)$ equals 1 at some stage $t>s_{0}$, so $\ell_{\alpha}(e, t)=1$. Since $x \in \bar{A}, \Phi_{e}^{D}(e)\left[s_{0}\right]=\Phi_{e}^{D}(e) \downarrow$ and both computations are the same. Since $e$ was chosen so that $\lim _{s} \ell_{\alpha}(e, s) \neq K^{D}(e)$, we know that $\ell_{\alpha}(e, s)$ must change back to 0, which can only happen in Step 5 . However, this can't happen since $\Phi_{e}^{D}(e)\left[s_{0}\right] \downarrow$ and never changes below the use. This gives a contradiction. Thus, if $S_{h, e}^{\alpha} \cap \bar{A}$ is nonempty, then $\lim _{s} h(r(\alpha, e, n), s)=0$.

Case 2: Suppose $S_{h, e}^{\alpha} \cap \bar{A}$ is empty.

First, note that if $S_{h, e}^{\alpha}$ has infinitely many witnesses, all of which enter $A$, then Step 5 acts infinitely often, meaning that $\Phi_{e}^{D}(e)[s]$ changes infinitely often, so $\Phi_{e}^{D}(e)$ diverges, and thus $\lim _{s} \ell_{\alpha}(e, s) \neq 0$. Since $\ell_{\alpha}(e, s)=1$ infinitely often, we must have that $h(r(\alpha, e, n), s)$ switches from 0 to 1 infinitely often, as it must be 0 when each witness is appointed and 1 when $\ell_{\alpha}(e, s)$ changes to 1 . Thus $\lim _{s} h(r(\alpha, e, n, s)$ doesn't exist.

We may thus assume that after a finite stage, $S_{h, e}^{\alpha} \cap \bar{A}$ is empty and never gets more elements. We may also assume that $\lim _{s} h(r(\alpha, e, n), s)=0$, else we have achieved our goal. We now show that $\mathrm{P}_{h, e}^{\alpha}$ will eventually be able to act by adding a new witness in Step 3, contradicting that $S_{h, e}^{\alpha}$ never gets a new element. We check that all the conditions of Step 3 will eventually be met.

For the first bullet point, we know that $\mathrm{P}_{h, e}^{\alpha}$ will be inactive for almost all $s$ since it is only active when $S_{h, e}^{\alpha} \cap \bar{A}$ is nonempty. Since $\mathrm{P}_{h, e}^{\alpha}$ is inactive, we know that $\ell_{\alpha}(e, s)=0$ at almost all stages, as it only can be 1 when $\mathrm{P}_{h, e}^{\alpha}$ is active. Now, we also know that $\lim _{s} \ell_{\alpha}(e, s) \neq K^{D}(e)$, so $\Phi_{e}^{D}(e) \downarrow$. This gives us the second bullet point for almost all $s$. By the assumption in the previous paragraph, for almost all $s, h(r(\alpha, e, n), s)=0$, so we meet the third bullet point.

To check that the fourth bullet point is met, note that for all $e^{\prime}<e$, either $K^{D}\left(e^{\prime}\right)=1=\lim _{s} \ell_{\alpha}\left(e^{\prime}, s\right)$, in which case for almost all $s, e^{\prime}$ does not prevent $e$ from acting, or $K^{D}\left(e^{\prime}\right)=0=\lim _{s} \ell_{\alpha}\left(e^{\prime}, s\right)$. In the latter case, there are two possibilities. If for almost all $s, \Phi_{e^{\prime}}^{D}\left(e^{\prime}\right)[s] \uparrow$, then for almost all $s, e^{\prime}$ does not prevent $e$ from acting. Otherwise, there are infinitely many stages such that $\Phi_{e^{\prime}}^{D}\left(e^{\prime}\right)[s]$ converges, but since $\Phi_{e^{\prime}}^{D}\left(e^{\prime}\right) \uparrow$, the computation changes infinitely often, so eventually the computation will have changed at least $e$ times. Thus, $e^{\prime}$ only prevents $e$ from adding a new witness for finitely many stages.

We have shown that for almost all $s$, the conditions in all the bullet points are met. We still must show that $\alpha$ is allowed to add a witness at a stage where it is able to act.

Claim: For every $\alpha$ on the true path, $\alpha$ is allowed to add witnesses infinitely often.

Proof of Claim: Induct on the length of $\alpha$. Assume true for the predecessor of $\alpha, \alpha^{-}$. Let $\alpha^{-}$have length $j$. If $\alpha(j)=1$, then $\alpha$ is allowed to act at every stage that $\alpha^{-}$is allowed to act. If $\alpha(j)=0$, we need to show that infinitely often, when $\alpha^{-}$is allowed to act, $\mathrm{L}_{j}$ allows action as well. Suppose not. Then there is some stage $s_{0}$ such that $\alpha$ is not allowed to act for any $s \geq s_{0}$. Each time $\alpha^{-}$is allowed to act after stage $s_{0}, \mathrm{~L}_{j}$ disallows $\alpha$ from adding new witnesses. This means that Step 2 does not apply for $j$ at any of these stages. We know 
that by the proof of Lemma 4.3, $\mathrm{L}_{j}$ disallows for each $N$ at most finitely often. Since $\alpha$ is on the true path, $W_{j}-\left(Y^{j} \cup A\right)$ has infinitely many expansionary stages, so $\mathrm{L}_{j}$ eventually disallows only for $N$ 's that Step 2 never acted for before stage $s_{0}$. Thus eventually Step 2 will apply to $j, N$, and $\alpha^{-}$, so $\alpha$ will be allowed to add new elements, which gives a contradiction, proving the claim.

Now wait until a stage $s_{0}$ such that the approximation to the true path never goes to the left of $\alpha$ and the bullet points do not prevent $\mathrm{P}_{h, e}^{\alpha}$ from acting. Let $s_{1}>s_{0}$ be the next $\alpha$-stage, and $s_{2} \geq s_{1}$ the next stage that $\alpha$ is allowed to add witnesses. Then $s_{2}$ is either an $\alpha$-stage or $\delta_{s_{2}}$ is to the right of $\alpha$, so we may perform Step 3 for $\mathrm{P}_{h}^{\alpha}$.

At infinitely many stages, Step 3 will be able to act by adding a witness. Thus, it will act, contradicting that no new witnesses are added. Therefore, if no new witnesses are ever added, then we must have that $\lim _{s} h(r(\alpha, e, n), s)$ does not equal 0 , satisfying the lemma.

Lemma 4.5. For each partial computable $\varphi_{i}=h, Y_{h} \cap \bar{A} \subseteq^{*} Z_{h}$.

Proof. Proving this lemma amounts to showing that there are only finitely many elements in $Y_{h} \cap \bar{A}$ that remain active. For each $e$ and each $\alpha$, there can only be one active element at a time. Let $e$ be such that $\mathrm{P}_{h, e}^{\alpha}$ is met, as in Lemma 4.4. Either $e$ resets all $e^{\prime}>e$ infinitely often or $\lim _{s} \ell_{\alpha}(e, s)$ exists.

If $\lim _{s} \ell_{\alpha}(e, s)=0$, then $\Phi_{e}^{D}(e) \downarrow$. Then $\Phi_{e}^{D}(e)[s]$ changes computations some finite number of times, so by the final bullet of Step 3, only finitely many $e^{\prime}>e$ will ever be able to add witnesses, and will only be able to do so finitely often. If $\lim _{s} \ell_{\alpha}(e, s)=1$, then $\Phi_{e}^{D}(e) \uparrow$, but this is not possible since $\ell_{\alpha}(e, s)=0$ if $\Phi_{e}^{D}(e)[s] \uparrow$.

Thus, for a fixed $\alpha$ and $h$, either almost all $\mathrm{P}_{h, e}^{\alpha}$ are reset infinitely often or only finitely many ever become active. Since there are only finitely many $\alpha$ of length $i, h=\varphi_{i}$, we conclude that $Y_{h} \cap \bar{A} \subseteq^{*} Z_{h}$.

Lemma 4.6. For each $j \in \omega, W_{j} \cap \bar{A}$ is infinite if and only if either the set of expansionary stages of $W_{j}-\left(Y^{j} \cup A\right)$ is infinite or $W_{j} \cap Z^{j}$ is infinite. Thus, $\bar{A}$ is semilow 1.5 .

Proof. This Lemma gives us that $\bar{A}$ is semilow ${ }_{1.5}$ because to tell if $W_{j} \cap \bar{A}$ is infinte, we can ask Inf if the c.e. set that is the union of $W_{j} \cap Z^{j}$ and the set of expansionary stages of $W_{j}-\left(Y^{j} \cup A\right)$ is infinite. This gives us an $m$-reduction from $W_{j} \cap \bar{A}$ to Inf.

First, note that $Z^{j} \subseteq \bar{A}$, so if $W_{j} \cap Z^{j}$ is infinite, so is $W_{j} \cap \bar{A}$. By Lemma 4.3. we know that if the set of expansionary stages of $W_{j}-\left(Y^{j} \cup A\right)$ is infinite, then $W_{j} \cap \bar{A}$ is infinite.

Assume $W_{j} \cap \bar{A}$ is infinite. Suppose $W_{j}-\left(Y^{j} \cup A\right)$ has finitely many expansionary stages. Then $W_{j}-\left(Y^{j} \cup A\right)$ is finite. Thus, $W_{j} \cap \bar{A} \subseteq^{*} W_{j} \cap Y^{j}$. By Lemma 4.5. $Y_{h} \cap \bar{A} \subseteq^{*} Z_{h}$, so $W_{j} \cap \bar{A} \subseteq^{*} W_{j} \cap Z^{j}$. Thus $W_{j} \cap Z^{j}$ is infinite.

Lemma 4.7. $A \leq_{\mathrm{T}} D$. 
Proof. To determine if $k \in D$, ask if any elements of the set $F_{k}$ are in $A$. The only way any of the elements can enter $A$ is if $k \in D$. It is always possible to enumerate one of the elements into $A$ because we must keep out at most

$\sum_{M=1}^{k} M=k(k+1) / 2$ elements, and $\frac{(k+1) k}{2}=\frac{k^{2}}{2}+\frac{k}{2}<2 k^{2}$, which is the size of $F_{k}$ for $k>0$. For $k=0$, we do not need to keep out any elements, and $F_{0}$ is nonempty.

Lemma 4.8. $D \leq_{\mathrm{T}} A$.

Proof. $\omega^{[0]} \cap A \leq_{\mathrm{T}} D$ since no element from $\omega^{[0]}$ enters $A$ unless it is in $F_{k}$ and $k$ enters $D$.

For $\omega-\omega^{[0]}, x \in A$ if and only if $x$ becomes a witness and then enters $A$. The set of witnesses is computable because if $x$ is not a witness by stage $x$, it will never become a witness. If $x$ is a witness, we ask what its use $u$ is as a witness. Let $s_{x}$ be such that $D\left\lceil u=D_{s_{x}}\left\lceil u\right.\right.$. Then $x \in A$ if and only if $x \in A_{s_{x}}$.

\section{Semilow $_{2}$, O.S.P, not semilow 1.5 in all nonlow degrees}

As mentioned previously, a set $B$ is semilow $w_{2}$ if $\left\{e \mid W_{e} \cap B\right.$ is infinite $\} \leq_{\mathrm{T}} \mathbf{0}^{\prime \prime}$. It follows immediately that if $B$ is semilow ${ }_{1.5}$, then $B$ is semilow s. $_{2}$

A set $A$ has the outer splitting property if there exist total computable functions $f$ and $g$ such that, for each $i \in \omega$,

(a) $W_{i}=W_{f(i)} \sqcup W_{g(i)}$,

(b) $W_{f(i)} \cap \bar{A}$ is finite, and

(c) if $W_{i} \cap \bar{A}$ is infinite, then $W_{f(i)} \cap \bar{A}$ is nonempty.

Maass [8] showed that if a set $A$ has semilow 1.5 complement, then $A$ has the outer splitting property. Thus, the class of c.e. sets with semilow ${ }_{1.5}$ complement

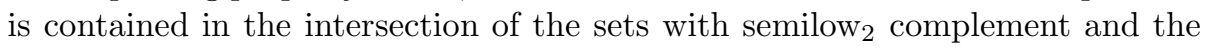
sets with the outer splitting property. We show that in every nonlow c.e. degree, this containment is strict.

Theorem 5.1. For every nonlow c.e. degree $\mathbf{d}$, there is a c.e. set $A \in \mathbf{d}$ such

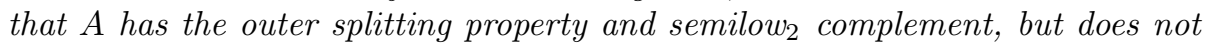
have semilow 1.5 complement.

Corollary 5.2. The nonlow c.e. degrees are precisely the degrees of c.e. sets that have the outer splitting property and semilow $w_{2}$ complement but not semilow $w_{1.5}$ complement. 
The corollary follows immediately from the theorem, since every low c.e. set has semilow 1.5 complement.

Proof. Given a nonlow c.e. set $D$, we will construct an $A \equiv_{\mathrm{T}} D$ such that $\bar{A}$ is semilow 2 but not semilow 1.5 and such that $A$ satisfies the outer splitting property.

\subsection{Requirements:}

To ensure that $D \leq_{\mathrm{T}} A$, we will code $D$ into $A$. To begin, we construct a computable list of disjoint finite sets $F_{k} \subset \omega^{[0]}$, such that $F_{0}$ contains two elements and each other $F_{k}$ has $6 k^{2}$ elements.

$\mathrm{C}_{k}: k \in D$ if and only if some element of $F_{k}$ is in $A$.

To make $\bar{A}$ not semilow 1.5 , we meet the following requirement for each total computable function $h$ :

$\mathrm{P}_{h}:\left\{e \mid W_{e} \cap \bar{A}\right.$ infinite $\} \neq\left\{e \mid W_{h(e)}\right.$ infinite $\}$.

Let $\left\{\varphi_{i}\right\}_{i \in \omega}$ be a listing of all partial computable functions on two inputs. Then we must meet $\mathrm{P}_{h}$ for all $h=\varphi_{i}$. As in Theorem 4.1 we find it easier to refer to $h$ instead of $i$ in this situation and when we say $h<j$, we mean that $i<j$.

We will try to meet $\mathrm{P}_{h}$ for each $\alpha$ of length $i$ for $h=\varphi_{i}$ on our tree of strategies and each $e \in \omega$, so we will label our requirements $\mathrm{P}_{h, e}^{\alpha}$. To meet $\mathrm{P}_{h}^{\alpha}$, for each $e$, if $\mathrm{P}_{h, e}^{\alpha}$ has been reset $n$ times, then we build $S_{h, e}^{\alpha}=W_{r(\alpha, e, n)}$, where $r$ is known in advance by the Recursion Theorem with Parameters. We add elements, called witnesses, to $S_{h, e}^{\alpha}$. For some $e$, we will guarantee that if $W_{h(r(\alpha, e, n))}$ is infinite, then we dump the witnesses into $A$, so $S_{h, e}^{\alpha} \cap \bar{A}$ is finite, and if $W_{h(r(\alpha, e, n))}$ is finite, $S_{h, e}^{\alpha}$ gets infinitely many witnesses that never enter $A$. Thus, $r(\alpha, e, n)$ will satisfy that $W_{r(\alpha, e, n)} \cap \bar{A}$ is infinite if and only if $W_{h(r(\alpha, e, n))}$ is finite.

Define

$Y_{h, e}^{\alpha}=\left\{x \mid x\right.$ is ever a witness for $\left.\mathrm{P}_{h, e}^{\alpha}\right\}$,

$Y_{h}=\left\{x \mid x\right.$ is in $Y_{h, e}^{\alpha}$ for some $e$ and $\left.\alpha\right\}$, and

$Y^{j}=\bigcup_{h \leq j} Y_{h}$.

Thus, $Y^{j}$ is the set of all $x$ that are ever witnesses for any $P_{h, e}^{\alpha}$ for any $h \leq j$.

To meet the outer splitting property, we build functions $f$ and $g$ so that for each $i$, we satisfy requirement $\mathrm{O}_{i}$ :

(a) $W_{i}=W_{f(i)} \sqcup W_{g(i)}$,

(b) $W_{f(i)} \cap \bar{A}$ is finite, and

(c) if $W_{i} \cap \bar{A}$ is infinite, then $W_{f(i)} \cap \bar{A}$ is nonempty. $0^{\prime \prime}$.

For $A$ to have semilow 2 complement, we need to ensure $\left\{j \mid W_{j} \cap \bar{A}\right.$ infinite $\} \leq_{\mathrm{T}}$

To achieve this, we will meet for each $j \in \omega$ : 
$\mathrm{L}_{j}: W_{j} \cap \bar{A}$ is infinite if and only if either $W_{j}-\left(Y^{j} \cup A\right)$ has infinitely many expansionary stages or $W_{j} \cap Y^{j} \cap \bar{A}$ is infinite.

We will also show that $\mathbf{0}^{\prime \prime}$ is able to determine if $W_{j} \cap Y^{j} \cap \bar{A}$ is infinite.

We use a binary tree of strategies. Let $\alpha$ be a length $i$ string on the tree. For each $j<i, \alpha(j)=0$ indicates a guess that $W_{j}-\left(Y^{j} \cup A\right)$ has infinitely many expansionary stages and $\alpha(j)=1$ is a guess that it has finitely many. $\mathrm{P}_{h}^{\alpha}$ will work to satisfy $\mathrm{P}_{h}$ while assuming that $\alpha$ is correctly guessing the true outcome.

The tree serves two purposes. As in Theorem 4.1, it allows us to ensure that if $W_{j}-\left(Y^{j} \cup A\right)$ has infinitely many expansionary stages, it is in fact an infinite set. The nodes guessing that there are infinitely many expansionary stages are not allowed to add witnesses when $\mathrm{L}_{j}$ is injured. Instead, they wait until a new expansionary stage is reached. This way, they do not create witnesses that may later enter $W_{j}-\left(Y^{j} \cup A\right)$, only to be forced into $A$. In addition, nodes guessing that $W_{j}-\left(Y^{j} \cup A\right)$ has finitely many expansionary stages are reset each time $W_{j}-\left(Y^{j} \cup A\right)$ reaches a new expansionary stage, meaning that any of their current witnesses will never enter $A$. The combination of these two actions guarantees that if $W_{j}-\left(Y^{j} \cup A\right)$ has infinitely many expansionary stages, it has infinitely many elements that never enter $A$ and so it is infinite.

The other purpose of the tree is to allow $\mathbf{0}^{\prime \prime}$ to compute whether $W_{j} \cap Y^{j} \cap \bar{A}$ is infinite. In Lemma 5.5 we analyze which elements of $Y^{j}$ stay in $\bar{A}$ forever by examining what happens to witnesses for $\mathrm{P}_{h}^{\alpha}$ when $\alpha$ has various relationships to the true path. Since $\mathbf{0}^{\prime \prime}$ can determine the true path, we can use $\mathbf{0}^{\prime \prime}$ to find the index for a c.e. set that is finitely different from $Y^{j} \cap \bar{A}$. Thus $\mathbf{0}^{\prime \prime}$ can determine whether $W_{j} \cap Y^{j} \cap \bar{A}$ is infinite.

In addition, we need that $A \leq_{\mathrm{T}} D$, which we achieve by a nonlow permitting argument, as in Theorem 4.1 .

\subsection{Construction}

We build an approximation $\delta_{s}$ to the true path. We call $s$ an $\alpha$-stage if $\alpha$ is a substring of $\delta_{s}$. Define $\delta_{0}=\lambda$, the empty string. Let $\delta_{s}$ be the length $s$ string defined by: $\delta_{s}(j)=0$ if $W_{j}-\left(Y^{j} \cup A\right)$ has reached a new expansionary stage since the last $\delta_{s}\lceil j$-stage, and 1 otherwise.

Let $\ell_{\alpha}(e, 0)=0$ and $\ell_{\alpha}(e, s+1)=\ell_{\alpha}(e, s)$ unless otherwise specified.

We begin with all $\mathrm{L}_{j}$ allowing addition of new witnesses for every $\mathrm{P}_{h}^{\alpha}$. In Step 5 , if $\mathrm{L}_{j}$ is injured, then it will disallow new witnesses for $\mathrm{P}_{h}^{\alpha}$, where $\alpha$ guesses the infinite outcome for $\mathrm{L}_{j} . \mathrm{L}_{j}$ will again allow new witnesses if $W_{j}-\left(Y^{j} \cup A\right)$ reaches a new expansionary stage (Step 1). Step 2 also has the ability to make $\mathrm{L}_{j}$ allow again, and serves the purpose of ensuring that if $W_{j}-\left(Y^{j} \cup A\right)$ has infinitely many expansionary stages, it allows each $\mathrm{P}_{h}^{\alpha}$ to add witnesses infinitely often.

Nodes on the tree may be put in 1-1 correspondence with $\mathbb{N}-\{0\}$ via a function $f: 2^{\in \omega} \rightarrow \mathbb{N}-\{0\}$. Using such a correspondence, let $\langle\alpha, e\rangle:=\langle f(\alpha), e\rangle$. In Step 3, witnesses come from $\omega^{[\langle\alpha, e\rangle]}$. 
Note that in the following, Steps 1 and 2 are identical to those in Theorem 4.1.

Stage $s+1$ :

Step 1 (new expansionary stage): For each $j \in \omega$, if $W_{j}-\left(Y^{j} \cup A\right)$ grows to a size we haven't seen before, i.e. it reaches a new expansionary stage, then do the following for each $\alpha$ of length greater than $j$ : If $\alpha$ guesses that $W_{j}-\left(Y^{j} \cup A\right)$ has infinitely many expansionary stages (i.e. $\alpha(j)=0$ ), then $\mathrm{L}_{j}$ now allows addition of new witnesses for $\mathrm{P}_{h}^{\alpha}$. If $\alpha$ guesses that $W_{j}-\left(Y^{j} \cup A\right)$ has finitely many expansionary stages $(\alpha(j)=1)$, we reset $\mathrm{P}_{h, e}^{\alpha}$ for all $e$, making $\ell_{\alpha}(e, s)=0$ and declaring $\mathrm{P}_{h, e}^{\alpha}$ and any witnesses for $\mathrm{P}_{h, e}^{\alpha}$ inactive. When reset, we will start a new $S_{h, e}^{\alpha}$ that begins empty.

Step 2 (allowing some disallowed witnesses): We say a node of length $j$ is allowed new witnesses if no $j^{\prime}<j$ disallows $\mathrm{P}_{h}^{\alpha}$ from adding witnesses. For each $j, N$, and $\beta$, where $\beta$ has length $j$, if $\mathrm{L}_{j}$ currently disallows for $N$ (as defined in Step 5) and if $\beta$ is allowed new witnesses, then set $\mathrm{L}_{j}$ to allow new witnesses for all $\alpha$ unless Step 2 has acted at some previous stage for these particular $j$, $N$, and $\beta$. Do this for all possible triples, of which there are only finitely many. This step guarantees that nodes on the true path will be allowed to act in Step 3 infinitely often.

Step 3 (adding witnesses): We act for each $\alpha$ of length at most $s$ such that $\mathrm{P}_{h}^{\alpha}$ is allowed to add witnesses by each $\mathrm{L}_{j}$ with $j<s$ such that $\alpha(j)=0$, and such that either $s$ is an $\alpha$-stage or $\alpha$ is to the left of $\delta_{s}$ and if $s_{\alpha}$ was the last $\alpha$-stage, $\mathrm{P}_{h}^{\alpha}$ has not been allowed to act at any stage $t, s_{\alpha} \leq t<s$. In other words, we act on the approximation to the true path $\delta_{s}$ and for any nodes to the left of $\delta_{s}$ that have not been allowed to act since before they were last on the approximation to the true path. We act in length-lexicographic order. For each such $\alpha$, we ask if there is any $e<s$ such that

- $\ell_{\alpha}(e, s)=0$,

- $\Phi_{e}^{D}(e)[s] \downarrow$, and

- for all $e^{\prime}<e, \Phi_{e^{\prime}}^{D}\left(e^{\prime}\right)[s] \downarrow \Longleftrightarrow \ell_{\alpha}\left(e^{\prime}, s\right)=1$ or $\Phi_{e^{\prime}}^{D}\left(e^{\prime}\right)[s] \downarrow$ and has changed computations at least $e$ times since the last time that $\mathrm{P}_{h, e}^{\alpha}$ added a witness.

If so, then for each such $e$, let $x>s, x \in \omega^{\langle\alpha, e\rangle}$, be a fresh witness, larger than any previously chosen witness. Enumerate $x \in S_{h, e}^{\alpha}$. Declare $x$ to have use $\varphi_{e}^{D}(e)[s]$, which is defined to be the use of $\Phi_{e}^{D}(e)[s]$. We call the witness $x$ active.

Step 4 (changing $\ell_{\alpha}$ when $W_{h(r(\alpha, e, n)}$ grows): Suppose $\ell_{\alpha}(e, s)=0, S_{h, e}^{\alpha} \cap \bar{A}$ contains at least one witness, and $\left|W_{h(r(\alpha, e, n), s+1}\right|>\left|W_{h(r(\alpha, e, n), s}\right|$ where $n$ is the number of times $\mathrm{P}_{h, e}^{\alpha}$ has been reset. Then set $\ell_{\alpha}(e, s+1)=1$ and reset $\mathrm{P}_{h, e^{\prime}}^{\alpha}$ for all $e^{\prime}>e$ by starting new empty sets $S_{h, e^{\prime}}^{\alpha}$, as well as setting $\ell_{\alpha}\left(e^{\prime}, s+1\right)=0$ and declaring any witnesses for $\mathrm{P}_{h, e^{\prime}}^{\alpha}$ inactive. Perform this step for each $\alpha$ and for the least $e$ such that it applies. 
Step 5 (enumerating witnesses into A and disallowing new witnesses): If $x \in S_{h, e}^{\alpha}$ is active with use $u$ and $D_{s+1}\left\lceil u \neq D_{s} \uparrow u\right.$, then put $x$ into $A_{s+1}$ and set $\ell_{\alpha}(e, s+1)=0$.

If by such an enumeration, we cause an element of $\left(W_{j}-\left(Y^{j} \cup A\right)\right)[s]$ to enter $A$, then we say $\mathrm{L}_{j}$ does not allow $\mathrm{P}_{h}^{\alpha}$ to add witnesses for any $\alpha$ such that $\alpha(j)=0$. In addition, if the size of $\left(W_{j}-\left(Y^{j} \cup A\right)\right)$ has become $N$, we say that $\mathrm{L}_{j}$ disallows for $N$.

Step 6 (outer splitting property): Let $X_{i}=\{x \mid x$ is ever a witness for any $\mathrm{P}_{h, e}^{\alpha}$ with $\left.\langle h, e\rangle<i\right\}$. For each $x \in W_{i, s+1}$ such that $x$ is not yet in $W_{f(i)}$ or $W_{g(i)}$, do the first of the following that applies:

(a) If $x \in W_{i}-\left(X_{i} \cup A\right)$ and $\left|W_{f(i)}-\left(X_{i} \cup A\right)\right|<i+1$, put $x$ in $W_{f(i)}$. Reset all $\mathrm{P}_{h, e}^{\alpha}$ for $\langle h, e\rangle \geq i$.

(b) If there is an $\langle h, e\rangle<i$ and any $\alpha$ such that $x$ is an inactive witness for $\mathrm{P}_{h, e}^{\alpha}$, and $W_{f(i)}$ does not already contain an inactive witness, then put $x$ into $W_{f(i)}$.

(c) If $W_{f(i)}$ contains no inactive witness and there is an $\langle h, e\rangle<i$ such that $x \in W_{i} \cap S_{h, e}^{\alpha}$ and $W_{f(i)} \cap S_{h, e}^{\alpha}$ is empty, then put $x$ into $W_{f(i)}$.

(d) If none of the above applies, put $x$ into $W_{g(i)}$.

Step 7 (coding D): If $k \in D_{s+1}-D_{s}$, enumerate one element from $F_{k}$ into $A_{s+1}$ such that it obeys the following: for each $M>0$ and $j+M=k$, the element is not one of the least $M$ elements in $\left(W_{j}-\left(Y^{j} \cup A\right)\right)[s]$, and for each $i \leq k$, the element is not one of the least $i+1$ elements in $W_{f(i)}-\left(X_{i} \cup A\right)$.

\subsection{Verification}

Lemma 5.3. For each $j \in \omega, W_{j} \cap \bar{A}$ is infinite if and only if either $W_{j} \cap \bar{A} \cap Y^{j}$ is infinite or $W_{j}-\left(Y^{j} \cup A\right)$ has infinitely many expansionary stages.

Proof. Note that the proof of this lemma is a very slight variation on the proof of Lemma 4.3 .

If $W_{j} \cap \bar{A}$ is infinite then either $W_{j} \cap \bar{A} \cap Y^{j}$ is infinite or $W_{j}-\left(Y^{j} \cup A\right)$ is infinite, in which case it has infinitely many expansionary stages.

If $W_{j} \cap \bar{A} \cap Y^{j}$ is infinite, then $W_{j} \cap \bar{A}$ is infinite. Suppose $W_{j}-\left(Y^{j} \cup A\right)$ has infinitely many expansionary stages. We will show that for each $M, W_{j}-\left(Y^{j} \cup A\right)$ has at least $M$ elements.

Induct on $M$. Assume true for $M-1$.

No element in $\left(W_{j}-\left(Y^{j} \cup A\right)\right)[s]$ ever enters $Y^{j}$ since new witnesses are chosen to be larger than $s$, and elements of $\left(W_{j}-\left(Y^{j} \cup A\right)\right)[s]$ are smaller than $s$.

For $k \leq j$, only finitely many elements are ever put into $A$ by $\mathrm{C}_{k}$. For $k>j$, $\mathrm{C}_{k}$ can only bring the size of $W_{j}-\left(Y^{j} \cup A\right)$ below $M$ if $M+j>k$, which happens finitely often. Let $s_{0}$ be a stage by which the least $M-1$ elements in $\left(W_{j}-\left(Y^{j} \cup A\right)\right)\left[s_{0}\right]$ never enter $A$ and after which no $\mathrm{C}_{k}$ enumerates any of the 
least $M$ elements of $\left(W_{j}-\left(Y^{j} \cup A\right)\right)[s]$ into $A$. Let $s_{1}>s_{0}$ be a stage after which Step 2 never acts for $(j, M-1, \beta)$ for any $\beta$ of length $j$. We may assume that at every stage $s>s_{1}$, the $M$ th least element of $\left(W_{j}-\left(Y^{j} \cup A\right)\right)[s]$ is a witness for some $\mathrm{P}_{h, e}^{\alpha}$, else it would never enter $A$ and we would be guaranteed to have at least $M$ elements in $W_{j}-\left(Y^{j} \cup A\right)$, as desired. Note that the length of $\alpha$ is greater than $j$ since the witnesses are not in $Y^{j}$.

Let $s_{2}>s_{1}$ be a stage such the $M$ th least element in $\left(W_{j}-\left(Y^{j} \cup A\right)\right)\left[s_{2}\right]$, called $x_{M}$, has the least use of any that will ever be in the $M$ th position of any $\left(W_{j}-\left(Y^{j} \cup A\right)\right)[s]$ for $s \geq s_{2}$. Now, if $x_{M}$ never enters $A$ by Step 5 , we are done, so assume $x_{M}$ enters $A$. When $x_{M}$ enters $A$, all witnesses with equal or larger uses also enter $A$, and since the next element in the $M$ th position is a witness, and it cannot have smaller use than $x_{M}$ due to minimality, then the next element that enters the $M$ th position has yet to become a witness. When $x_{M}$ enters $A, \mathrm{~L}_{j}$ does not allow any $\mathrm{P}_{h}^{\alpha}$ to add witnesses for any $\alpha$ such that $\alpha(j)=0$. Step 2 has finished acting for $j$ and $M-1$, so it will not cause $\mathrm{L}_{j}$ to later allow. The next time $\mathrm{L}_{j}$ allows will be by Step 1, which means $\left(W_{j}-\left(Y^{j} \cup A\right)\right)$ has already reached a new expansionary stage. Thus, at the first expansionary stage after $x_{M}$ enters $A$, the only witness that could be in the $M$ th position must be a witness for an $\alpha$ with $\alpha(j)=1$. At that new expansionary stage, Step 1 inactivates the witnesses for $\alpha$. Thus the element in the $M$ th position can never enter $A$, so $\left(W_{j}-\left(Y^{j} \cup A\right)\right)$ has at least $M$ elements.

We say that $\alpha$ is on the true path if $\alpha(j)=0$ if and only if $W_{j}-\left(Y^{j} \cap \bar{A}\right)$ has infinitely many expansionary stages during the construction.

Lemma 5.4. Let $\alpha$ be on the true path. Let e be the least such that either $\lim _{s} \ell_{\alpha}(e, s)$ does not exist or it exists and $\lim _{s} \ell_{\alpha}(e, s) \neq K^{D}(e)$. Then $P_{h, e}^{\alpha}$ is reset finitely often ( $n$ times) and $W_{h(r(\alpha, e, n))}$ is infinite if and only if $W_{r(\alpha, e, n)} \cap$ $\bar{A}$ is finite. Thus $\bar{A}$ is not semilow 1.5 .

Proof. Since $\alpha$ is on the true path, $\mathrm{P}_{h, e}^{\alpha}$ is reset only finitely often by $\mathrm{L}_{j}, j<h$. $\mathrm{P}_{h, e}^{\alpha}$ can only be reset by $\mathrm{O}_{i}$ in Step 6 when $\langle h, e\rangle \geq i$, and each of these $\mathrm{O}_{i}$ can only reset it finitely often, so it only gets reset finitely often by $\mathrm{O}_{i}$.

$\mathrm{P}_{h, e}^{\alpha}$ can only be reset by $\mathrm{P}_{h, e^{\prime}}^{\alpha}$ for $e^{\prime}<e$ when $W_{h\left(r\left(\alpha, e^{\prime}, n^{\prime}\right)\right)}$ grows, where $n^{\prime}$ is the number of times $\mathrm{P}_{h, e^{\prime}}^{\alpha}$ has been reset. When it grows, we set $\ell_{\alpha}\left(e^{\prime}, s+1\right)=1$ from $\ell_{\alpha}\left(e^{\prime}, s\right)=0$, so if $\mathrm{P}_{h, e^{\prime}}^{\alpha}$ resets $\mathrm{P}_{h, e}^{\alpha}$ infinitely often, then $\lim _{s} \ell_{\alpha}\left(e^{\prime}, s\right)$ does not exist, contradicting the assumption that it exists and equals $K^{D}\left(e^{\prime}\right)$. Thus $\mathrm{P}_{h, e}^{\alpha}$ is reset finitely often.

Case 1: $W_{h(r(\alpha, e, n))}$ is infinite.

Case 1a: $\Phi_{e}^{D}(e)$ diverges. Thus, $\lim _{s} \ell_{\alpha}(e, s) \neq 0$. If $S_{h, e}^{\alpha}$ is finite, we're done, since $W_{r(\alpha, e, n)}=S_{h, e}^{\alpha}$. If $S_{h, e}^{\alpha}$ is infinite, then $\Phi_{e}^{D}(e)[s]$ converges infinitely often. Any elements that we add when $\Phi_{e}^{D}(e)[s]$ converges will be enumerated into $A$ when $\Phi_{e}^{D}(e)[t]$ diverges for $t>s$ because the use of each witness equals the use of the computation $\Phi_{e}^{D}(e)[s]$. Thus $W_{r(\alpha, e, n)} \cap \bar{A}=S_{h, e}^{\alpha} \cap \bar{A}$ is finite. 
Case 1b: $\Phi_{e}^{D}(e)$ converges. Thus, $\lim _{s} \ell_{\alpha}(e, s) \neq 1$. If $W_{r(\alpha, e, n)} \cap \bar{A}$ is infinite, then $\lim _{s} \ell_{\alpha}(e, s)$ cannot equal 0 because there will be infinitely many stages where Step 4 applies, setting $\ell_{\alpha}(e, s+1)$ to 1 . Thus, if $\Phi_{e}^{D}(e) \downarrow$, then $\lim _{s} \ell_{\alpha}(e, s)$ does not exist, but this is also impossible since the only time that $\ell_{\alpha}(e, s)$ changes from 1 to 0 is when $\Phi_{e}^{D}(e)[s]$ diverges or changes its computation, meaning that $\Phi_{e}^{D}(e)$ must diverge.

Thus, if $W_{h(r(\alpha, e, n))}$ is infinite, then $W_{r(\alpha, e, n)} \cap \bar{A}$ is finite.

Case 2: $W_{h(r(\alpha, e, n))}$ is finite. Note that $\lim _{s} \ell_{\alpha}(e, s)$ must exist because it can change to 1 only finitely often since $W_{h(r(\alpha, e, n))}$ is finite.

Case 2a: $\Phi_{e}^{D}(e)$ diverges. Thus, $\lim _{s} \ell_{\alpha}(e, s)=1$. Note that at the final stage $s$ when $\ell_{\alpha}(e, s)$ changes from 0 to $1, S_{h, e}^{\alpha}$ contains an active witness not in $A$. This means that $\Phi_{e}^{D}(e)[s] \downarrow$, else the active witness would have entered $A$. Since $\Phi_{e}^{D}(e) \uparrow$, then the computation $\Phi_{e}^{D}(e)[s]$ must eventually be injured, at which point $\ell_{\alpha}(e, t)$ will become 0 , contradicting that the limit is 1 .

Case 2b: $\Phi_{e}^{D}(e)$ converges. Thus, $\lim _{s} \ell_{\alpha}(e, s)=0$. Because $\Phi_{e}^{D}(e)$ converges, if infinitely many elements enter $S_{h, e}^{\alpha}$, then infinitely many elements stay in $S_{h, e}^{\alpha} \cap \bar{A}$, since they only enter if $\Phi_{e}^{D}(e)[s]$ has its computation injured. Thus it suffices to show that infinitely elements enter $S_{h, e}^{\alpha}$. To do so, we will show that at infinitely many stages, Step 3 acts. We already know that for almost all $s, \ell_{\alpha}(e, s)=0$ and $\Phi_{e}^{D}(e)[s] \downarrow$, so the first two bullet points of Step 3 are met for almost all $s$.

To check that the third bullet point is met, note that for each $e^{\prime}<e$, either $K^{D}\left(e^{\prime}\right)=1=\lim _{s} \ell_{\alpha}\left(e^{\prime}, s\right)$, in which case for almost all $s, e^{\prime}$ does not prevent $e$ from acting, or $K^{D}\left(e^{\prime}\right)=0=\lim _{s} \ell_{\alpha}\left(e^{\prime}, s\right)$. In the latter case, there are two possibilities. If for almost all $s, \Phi_{e^{\prime}}^{D}\left(e^{\prime}\right)[s] \uparrow$, then for almost all $s, e^{\prime}$ does not prevent $e$ from meeting the third bullet point. Otherwise, there are infinitely many stages such that $\Phi_{e^{\prime}}^{D}\left(e^{\prime}\right)[s] \downarrow$, but since $\Phi_{e^{\prime}}^{D}\left(e^{\prime}\right) \uparrow$, the computation changes infinitely often, so eventually the computation will have changed at least $e$ times. Thus, $e^{\prime}$ only prevents $e$ from adding a new witness for finitely many stages.

Now that we have seen that for almost all $s$, the three bullet points are met, we must still show that $\alpha$ is allowed to add a witness at a stage where it is able to act.

Claim: For every $\alpha$ on the true path, $\alpha$ is allowed to add witnesses infinitely often.

For the proof of the claim, see Lemma 4.4 replacing the reference to Lemma 4.3 with Lemma 5.3 .

Now wait until a stage $s_{0}$ such that the approximation to the true path never goes to the left of $\alpha$ and the bullet points do not prevent $\mathrm{P}_{h, e}^{\alpha}$ from acting after stage $s_{0}$. Let $s_{1}>s_{0}$ be any $\alpha$-stage, and $s_{2} \geq s_{1}$ the next stage that $\alpha$ is allowed to add witnesses. Then $s_{2}$ is either an $\alpha$-stage or $\delta_{s_{2}}$ is to the right of $\alpha$, so we may perform Step 3 for $\mathrm{P}_{h, e}^{\alpha}$. Thus, there are infinitely many stages at which we will add witnesses to $S_{h, e}^{\alpha}$, so it will be infinite and have infinite intersection with $\bar{A}$.

Lemma 5.5. $0^{\prime \prime}$ can compute $\left\{j \mid W_{j} \cap \bar{A}\right.$ is infinite $\}$. 
Proof. By Lemma 5.3, it suffices to show that $\mathbf{0}^{\prime \prime}$ can compute $\left\{j \mid W_{j} \cap \bar{A} \cap Y^{j}\right.$ is infinite $\}$, since $\mathbf{0}^{\prime \prime}$ can determine if the set of expansionary stages of $W_{j}-\left(Y^{j} \cup A\right)$ is infinite since the set of expansionary stages is a c.e. set.

We will show that $\bar{A} \cap Y^{j}$ is a c.e. set and that there is a $\mathbf{0}^{\prime \prime}$-computable function $m(j)$ such that $W_{m(j)}={ }^{*} \bar{A} \cap Y^{j}$, so $W_{j} \cap \bar{A} \cap Y^{j}$ is infinite if and only if $W_{j} \cap W_{m(j)}$ is infinite, which is $\mathbf{0}^{\prime \prime}$-computable.

Note that $\mathbf{0}^{\prime \prime}$ can determine the true path on the tree of strategies. Consider each $Y_{h, e}^{\alpha}$ that makes up $Y^{j}$. If $\alpha$ is to the right of the true path, then $\mathrm{P}_{h, e}^{\alpha}$ gets reset infinitely often, so the set of elements in $Y_{h, e}^{\alpha} \cap \bar{A}$ is the c.e. set given by the set of all $x$ in $\mathrm{Y}_{h, e}^{\alpha} \cap \bar{A}$ at a stage when $\mathrm{P}_{h, e}^{\alpha}$ is reset. Put these into $W_{m(j)}$.

If $\alpha$ is to the left of the true path, there are only finitely many $\alpha$-stages. Step 3 is either allowed to add witnesses at the final $\alpha$-stage or at one later stage. Thus $S_{h, e}^{\alpha}$ only gets finitely many elements, so it may be ignored.

For each $\alpha$ on the true path of length $i \leq j$, use $\mathbf{0}^{\prime \prime}$ to find the least $e$ such that $\lim _{s} \ell_{\alpha}(e, s) \neq K^{D}(e)$. For $e^{\prime}<e$, note that if there are infinitely many witnesses for $\mathrm{P}_{h, e^{\prime}}^{\alpha}$, then $\ell_{\alpha}\left(e^{\prime}, s\right)$ must be 0 infinitely often, so its limit must be 0 . We also have that $\Phi_{e^{\prime}}^{D}\left(e^{\prime}\right)[s]$ must converge infinitely often for us to add infinitely many witnesses, so it must also diverge infinitely often so that $K^{D}\left(e^{\prime}\right)=\lim _{s} \ell_{\alpha}\left(e^{\prime}, s\right)=0$. Thus, once $\mathrm{P}_{h, e^{\prime}}^{\alpha}$ stops being reset, which we know must happen by the proof of Lemma 5.4, all new witnesses eventually enter $A$. Thus, $\mathrm{P}_{h, e^{\prime}}^{\alpha}$ only contributes finitely much to $Y^{j} \cap \bar{A}$ and can be ignored.

Now consider $e^{\prime}>e$. If $\mathrm{P}_{h, e}^{\alpha}$ resets $\mathrm{P}_{h, e^{\prime}}^{\alpha}$ infinitely often, then the contribution of $\mathrm{P}_{h, e^{\prime}}^{\alpha}$ to $Y^{j} \cap \bar{A}$ is given by the set of elements that are witnesses at stages when it gets reset, and these can be added to $W_{m(j)}$. Suppose $\mathrm{P}_{h, e}^{\alpha}$ resets $\mathrm{P}_{h, e^{\prime}}^{\alpha}$ only finitely often. First, consider the case where $\lim _{s} \ell_{\alpha}(e, s)=1$. Then $\Phi_{e}^{D}(e) \uparrow$ by the choice of $e$. However, if $\lim _{s} \ell_{\alpha}(e, s)=1$, then when $\ell_{\alpha}(e, s)$ gets defined as 1 , it contains an active witness, and the witness cannot enter $A$ else it would reset $e^{\prime}$, so $\Phi_{e}^{D}(e) \downarrow$. This is a contradiction, so $\lim _{s} \ell_{\alpha}(e, s) \neq 1$. Since $\mathrm{P}_{h, e}^{\alpha}$ resets $\mathrm{P}_{h, e^{\prime}}^{\alpha}$ only finitely often, we must have that $\lim _{s} \ell_{\alpha}(e, s)=0$. Thus, $\Phi_{e}^{D}(e) \downarrow$, so $\Phi_{e}^{D}(e)[s]$ changes computations some finite number of times. By the final bullet of Step 3, only finitely many $e^{\prime}>e$ will ever be able to add witnesses, and will only be able to do so finitely often. Thus after a finite stage, no $\mathrm{P}_{h, e^{\prime}}^{\alpha}$ can add any witnesses, for $e^{\prime}>e$, so these collectively contribute only finitely many elements to $Y^{j} \cap \bar{A}$ and can be ignored.

For $e$ itself, $S_{h, e}^{\alpha} \cap \bar{A}$ is infinite if and only if $W_{h(r(\alpha, e, n))}$ is finite, for $n$ the number of times $\mathrm{P}_{h, e}^{\alpha}$ is reset, by Lemma 5.4. We can ask $\mathbf{0}^{\prime \prime}$ to determine this. If we know that $S_{h, e}^{\alpha} \cap \bar{A}$ is finite, we can ignore it. If we know it is infinite, then

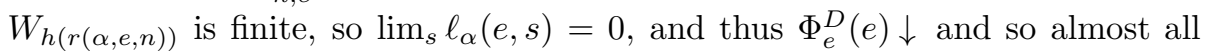
elements that we put into $S_{h, e}^{\alpha}$ remain in $\bar{A}$, so we can put them all into $W_{m(j)}$.

Lemma 5.6. The following hold for all $i \in \omega$ :

(a) $W_{i}=W_{f(i)} \sqcup W_{g(i)}$,

(b) $W_{f(i)} \cap \bar{A}=* \emptyset$, and 
(c) $W_{i} \cap \bar{A}$ infinite $\Longrightarrow W_{f(i)} \cap \bar{A}$ is nonempty.

Proof. By construction, we put every element of $W_{i}$ into either $W_{f(i)}$ or $W_{g(i)}$, but not both, so part (a) holds.

Proof of $(b)$ : We may add elements to $W_{f(i)}$ by Steps $6 \mathrm{a}, 6 \mathrm{~b}$, and $6 \mathrm{c}$. The set $\left(W_{f(i)} \cap \bar{A}\right)-X_{i}=W_{f(i)}-\left(X_{i} \cup A\right)$ must be finite because only Step 6a can add elements to it and only when its size is less than $i+1$, so at most $i+1$ elements will stay in $W_{f(i)}-\left(X_{i} \cup A\right)$ forever.

To see that $W_{f(i)} \cap \bar{A} \cap X_{i}$ is finite, we look at Steps $6 \mathrm{~b}$ and 6c. If Step $6 \mathrm{~b}$ adds an inactive witness to $W_{f(i)}$, it will never be able to do so again, as inactive witnesses remain inactive witnesses forever for the remainder of the construction. Step 6c allows us to take an element from $W_{i} \cap S_{h, e}^{\alpha} \cap \bar{A}$ only when there is not already an element in $W_{f(i)} \cap S_{h, e}^{\alpha} \cap \bar{A}$, for $\langle h, e\rangle<i$. Thus, even if we put infinitely many elements in by Step $6 c$, only finitely many remain forever.

Proof of (c): Suppose $W_{i} \cap \bar{A}$ is infinite. Suppose there are eventually $i+1$ elements that enter $W_{f(i)}$ by Step 6a. When we add elements by Step 6a, they are taken from $W_{i}-\left(X_{i} \cup A\right)$, which means that they cannot be put into $A$ by action of $\mathrm{P}_{h, e}^{\alpha}$ for any $\langle h, e\rangle<i$. When we put the elements into $W_{f(i)}$, we also reset $\mathrm{P}_{h, e}^{\alpha}$ for each $\langle h, e\rangle \geq i$, so they cannot enter $A$ from action of these requirements, either. They can only be enumerated into $A$ by $\mathrm{C}_{k}$, for $k<i$, which each only act once. Thus, at least one element will remain in $W_{f(i)} \cap \bar{A}$. If there are not eventually $i+1$ elements that enter $W_{f(i)}$ by Step 6a, then $W_{i}-\left(X_{i} \cup A\right)$ must be finite, so $W_{i} \cap \bar{A} \subseteq^{*} X_{i}$, and so $W_{i} \cap \bar{A} \cap X_{i}$ is infinite. If Step 6b ever acts by enumerating into $W_{f(i)}$, then $W_{f(i)}$ contains an inactive witness, which will never enter $A$. Otherwise, we know there is some $\langle h, e\rangle<i$ such that there are infinitely many witnesses for $\mathrm{P}_{h, e}^{\alpha}$ contained in $W_{i} \cap \bar{A}$, by the pigeonhole principle. Since Step $6 \mathrm{~b}$ never acts, all of the witnesses are active when they enter $W_{i}$. Suppose $W_{f(i)} \cap \bar{A}$ is empty. Then every time Step 6c acts by adding an active witness to $W_{f(i)}$, that element later enters $A$ when the current computation $\Phi_{e}^{D}(e)[s]$ changes because $D$ changes below the use. For each $x$ that is an active witness for $\mathrm{P}_{h, e}^{\alpha}$ when it enters $W_{i}$, it either enters $W_{f(i)}$, in which case it later enters $A$, or there is already an active witness $y$ for $\mathrm{P}_{h, e}^{\alpha}$ in $W_{f(i)} \cap \bar{A}$ when it enters $W_{i}$. In that case, we know that $y$ goes into $A$, at which stage $x$ must go into $A$ as well because they have the same use. Thus, if $W_{f(i)} \cap \bar{A}$ is empty, then $W_{i} \cap \bar{A}$ is finite, proving part (c).

Lemma 5.7. $D \leq_{\mathrm{T}} A$.

Proof. To determine if $k \in D$, ask if $A$ contains any elements of $F_{k} . F_{k} \cap A$ is nonempty if and only if $k \in D$. This is because only $\mathrm{C}_{k}$ can enumerate elements of $F_{k}$ into $A$, and $\mathrm{C}_{k}$ is always able to act because $F_{k}$ has more elements in it than are prohibited. To see that $F_{k}$ has more elements than are prohibited, recall that for each $M>0$ and $j+M=k$, we prohibit the least $M$ elements in $\left(W_{j}-\left(Y^{j} \cup A\right)\right)[s]$, and for each $i \leq k$, we prohibit the least $i+1$ elements in $W_{f(i)}-\left(X_{i} \cup A\right)$. For $k=0$, one element is prohibited and $F_{0}$ has two 
elements. For $k>0$, the first clause prohibits less than $2 k^{2}$ elements because $\sum_{M=1}^{k} M=\frac{(k+1) k}{2}=\frac{k^{2}}{2}+\frac{k}{2}<2 k^{2}$. The second clause prohibits less than $4 k^{2}$ elements because $\sum_{i=0}^{k} i+1=\frac{(k+1)(k+2)}{2}=\frac{k^{2}}{2}+\frac{3 k}{2}+1<4 k^{2}$. Thus fewer than $6 k^{2}$ elements are prohibited and $F_{k}$ contains $6 k^{2}$ elements.

Lemma 5.8. $A \leq_{\mathrm{T}} D$.

Proof. $\omega^{[0]} \cap A \leq_{\mathrm{T}} D$ since no element from $\omega^{[0]}$ enters $A$ unless it is in $F_{k}$ and $k$ enters $D$.

For $\omega-\omega^{[0]}, x \in A$ if and only if $x$ becomes a witness and then enters $A$. The set of witnesses is computable because if $x$ is not a witness by stage $x$, it will never become a witness. If $x$ is a witness, we ask what its use $u$ is as a witness. Let $s_{x}$ be such that $D\left\lceil u=D_{s_{x}}\left\lceil u\right.\right.$. Then $x \in A$ if and only if $x \in A_{s_{x}}$.

This concludes the proof of the theorem.

\section{Non-Low 2 degrees and sets whose complement is not semilow 2}

In this section we will provide an index set argument that every nonlow 2 c.e degree contains a c.e. set whose complement is not semilow 2 . Soare [13, IV 4.11] shows via an index set argument that every non-low c.e degree contains a c.e. set whose complement is not semilow.

Recall that Inf is the set of indices for infinite c.e. sets. $\operatorname{Inf} f^{B}$ is $\Pi_{2}^{0}$ complete. $\operatorname{Inf} f^{B}=\left\{e \mid W_{e}^{B}\right.$ is infinite $\}$ and $\overline{B^{\prime \prime}} \leq_{1} \operatorname{Inf}{ }^{B}$. Let $\operatorname{Inf}(X)=\left\{e \mid W_{e} \cap X\right.$ is infinite $\}$. Recall $\bar{A}$ is semilow ${ }_{1.5}$ if $\operatorname{Inf}(\bar{A}) \leq_{1}$ Inf and $\bar{A}$ is semilow 2 if $\operatorname{Inf}(\bar{A}) \leq_{T} \operatorname{Inf}$

Theorem 6.1. For every c.e. set $B$, there is a c.e. $A \equiv_{\mathrm{T}} B$ such that $\operatorname{Inf}^{B} \leq_{1}$ $\operatorname{Inf}(\bar{A})$.

Corollary 6.2. If $B$ is nonlow n $_{2}$, then there exists a c.e. $A \equiv_{\mathrm{T}} B$ such that $\bar{A}$ is not semilow 2 (and not semilow 1.5 ).

Proof. Let $B$ be nonlow 2 . Then there exists a c.e. $A \equiv_{\mathrm{T}} B$ such that $\operatorname{Inf}<_{T}$ $\operatorname{Inf}^{B} \leq_{1} \operatorname{Inf}(\bar{A})$, so $\bar{A}$ is not semilow 2 (and not semilow ${ }_{1.5}$ ).

Proof of Theorem 6.1. Let $\left\{\Phi_{i}\right\}_{i \in \omega}$ be a listing of all Turing functionals. Let $\left\{B_{s}\right\}_{s \in \omega}$ be a computable enumeration of $B$. We may assume that if $\Phi_{i}^{B}(x)[s] \downarrow$ $\neq \Phi_{i}^{B}(x)[t] \downarrow$, then there is a stage between $s$ and $t$ such that the computation diverges. We may also assume that at each stage $s$, there is at most one pair $\langle i, x\rangle$ such that $\Phi_{i}^{B}(x)[s] \downarrow$ and $\Phi_{i}^{B}(x)[s-1] \uparrow$. The use function of the computation 
$\Phi_{i}^{B}(x)[s]$ is denoted $\varphi_{i}^{B}(x)[s]$ and is the maximal element of $B_{i, s}$ seen in the computation.

Construction:

Stage $s+1$ :

Step 1: If $b \in B_{s+1}-B_{s}$, enumerate all marked elements into $A_{s+1}$ whose markers have uses $u \geq b$. Whenever an element is enumerated into $A$, its marker is removed.

Step 2: If $\Phi_{i}^{B}(x)[s+1] \downarrow$, and there is no current marker $M_{\langle i, x\rangle}$, then choose the least element $y \in \bar{A}_{s}, y>s+1$, without a marker and place marker $M_{\langle i, x\rangle}$ on it. The use of this marker is $\varphi_{i}^{B}(x)[s+1]$.

Step 3: Let $b \notin B_{s+1}$ be the least such that there is no current marker $\Gamma_{b}$. Place $\Gamma_{b}$ on the least $y \in \bar{A}_{s}, y>s+1$, without any marker, and let $b$ be the use of this marker.

End construction.

Lemma 6.3. $A \leq_{\mathrm{T}} B$.

Proof. To determine if $y \in A$, first run the construction until stage $y$ to see if $y$ ever has a marker. If not, then $y \notin A$. If so, then if the marker was added at stage $s$ and the use of the marker on $y$ is $u$, ask if $B$ ever changes at or below $u$ after stage $s$. If so, then $y \in A$. Otherwise $y \notin A$.

Lemma 6.4. $B \leq_{\mathrm{T}} A$.

Proof. Recall that the use of any marker $\Gamma_{b}$ is $b$. Thus, once $B_{s}$ has settled up through $b$, no current or future marker $\Gamma_{b}$ will ever enter $A$. To determine if $b \in B$, run the construction until either $\Gamma_{b}$ is placed on an element in $\bar{A}$, in which case $b \notin B$, or $b$ enters $B_{s}$. If $b \notin B$, then eventually a marker $\Gamma_{b}$ will appear in Step 3 on an element in $\bar{A}$, as desired.

Lemma 6.5. Let $W_{f(i)}=\left\{y \mid(\exists s)(\exists x)\left[M_{\langle i, x\rangle}\right.\right.$ is on $y$ at stage $\left.\left.s\right]\right\} . W_{i}^{B}$ is infinite if and only if $W_{f(i)} \cap \bar{A}$ is infinite.

Proof. If $W_{i}^{B}$ is infinite, then there exist infinitely many $x$ such that $\Phi_{i}^{B}(x) \downarrow$. For such $x$, for almost all $s, \Phi_{i}^{B}(x)[s] \downarrow$ and $B_{s}$ never later changes at or below the use $\varphi_{i}^{B}(x)[s]$. For each such $x, M_{\langle i, x\rangle}$ will be undefined before the final computation $\Phi_{i}^{B}(x)[s]$ converges, because any previous computation would have been injured below the use, causing the marker to be removed. When the final computation appears, the marker will be placed by Step 2 on an element in $\bar{A}_{s}$, and this element will never enter $A$ since nothing enters $B$ at or below the use. Thus for each of these infinitely many $x$ values, the final resting place of $M_{\langle i, x\rangle}$ is in $\bar{A}$, and thus $W_{f(i)} \cap \bar{A}$ is infinite.

If $W_{i}^{B}$ is finite, then for almost all $x, \Phi_{i}^{B}(x)$ diverges, which means that for almost all $x$, every computation $\Phi_{i}^{B}(x)[s]$ that converges is injured below the use. Thus, for almost all $x$, whenever $M_{\langle i, x\rangle}$ is placed on an element, that element later enters $A$. There are only finitely many $x$ values where $M_{\langle i, x\rangle}$ is ever on an element that remains outside of $A$, and once a marker is placed on 
an element outside $A$, it remains there forever, and there is always only one current $M_{\langle i, x\rangle}$. Thus, only finitely many elements of $W_{f(i)}$ are in $\bar{A}$.

\section{References}

[1] Peter Cholak. Automorphisms of the lattice of recursively enumerable sets. Mem. Amer. Math. Soc., 113(541):viii+151, 1995.

[2] Peter Cholak and Leo A. Harrington. On the definability of the double jump in the computably enumerable sets. J. Math. Log., 2(2):261-296, 2002.

[3] Rodney G. Downey, Carl G. Jockusch, Jr., and Paul E. Schupp. Asymptotic density and computably enumerable sets. J. Math. Log., 13(2):1350005, 43, 2013.

[4] Rachel Epstein. The nonlow computably enumerable degrees are not invariant in $\mathcal{E}$. Trans. Amer. Math. Soc., 365(3):1305-1345, 2013.

[5] Leo Harrington and Robert I. Soare. Definable properties of the computably enumerable sets. Ann. Pure Appl. Logic, 94(1-3):97-125, 1998. Conference on Computability Theory (Oberwolfach, 1996).

[6] Leo A. Harrington and Robert I. Soare. The $\Delta_{3}^{0}$-automorphism method and noninvariant classes of degrees. J. Amer. Math. Soc., 9(3):617-666, 1996.

[7] A. H. Lachlan. Degrees of recursively enumerable sets which have no maximal supersets. J. Symbolic Logic, 33:431-443, 1968.

[8] W. Maass. Characterization of recursively enumerable sets with supersets effectively isomorphic to all recursively enumerable sets. Trans. Amer. Math. Soc., 279:311-336, 1983.

[9] Russell Miller. Orbits of computably enumerable sets: low sets can avoid an upper cone. Ann. Pure Appl. Logic, 118(1-2):61-85, 2002.

[10] Joseph R. Shoenfield. Degrees of classes of recursively enumerable sets. J. Symbolic Logic, 41:695-696, 1976.

[11] Robert I. Soare. Automorphisms of the lattice of recursively enumerable sets I: maximal sets. Ann. of Math. (2), 100:80-120, 1974.

[12] Robert I. Soare. Automorphisms of the lattice of recursively enumerable sets II: low sets. Ann. Math. Logic, 22:69-107, 1982.

[13] Robert I. Soare. Recursively Enumerable Sets and Degrees. Perspectives in Mathematical Logic, Omega Series. Springer-Verlag, Heidelberg, 1987. 